Swarthmore College

Works

7-7-2004

\title{
Collision-Induced Non-Adiabatic Transitions Between The Ion-Pair States Of Molecular lodine: A Challenge For Experiment And Theory
}

T. V. Tscherbul

A. A. Buchachenko

M. E. Akopyan

S. A. Poretsky

A. M. Pravilov

See next page for additional authors

Follow this and additional works at: https://works.swarthmore.edu/fac-chemistry

Part of the Physical Chemistry Commons

Let us know how access to these works benefits you

\section{Recommended Citation}

T. V. Tscherbul, A. A. Buchachenko, M. E. Akopyan, S. A. Poretsky, A. M. Pravilov, and Thomas Alex Stephenson. (2004). "Collision-Induced Non-Adiabatic Transitions Between The Ion-Pair States Of Molecular lodine: A Challenge For Experiment And Theory". Physical Chemistry Chemical Physics. Volume 6, Issue 13. 3201-3214. DOI: 10.1039/b402655a https://works.swarthmore.edu/fac-chemistry/17

This work is brought to you for free by Swarthmore College Libraries' Works. It has been accepted for inclusion in Chemistry \& Biochemistry Faculty Works by an authorized administrator of Works. For more information, please contact myworks@swarthmore.edu. 


\section{Authors}

T. V. Tscherbul, A. A. Buchachenko, M. E. Akopyan, S. A. Poretsky, A. M. Pravilov, and Thomas Alex Stephenson 


\title{
Collision-induced non-adiabatic transitions between the ion-pair states of molecular iodine: A challenge for experiment and theory
}

\author{
Timur V. Tscherbul, ${ }^{a}$ Alexei A. Buchachenko, ${ }^{* a}$ Mikhail E. Akopyan, ${ }^{b}$ Sergey A. Poretsky, \\ Anatoly M. Pravilov ${ }^{b}$ and Thomas A. Stephenson ${ }^{c}$ \\ ${ }^{a}$ Laboratory of Molecular Structure and Quantum Mechanics, Department of Chemistry, \\ Moscow State University, Moscow 119992, Russia.E-mail: alexei@classic.chem.msu.su; \\ Fax: +7 09593924 13; Tel: +7 0959392286 \\ ${ }^{b}$ V. A. Fock Institute of Physics, St. Petersburg State University, Ul'yanovskaya 1, \\ Staryj Peterhof, St. Petersburg 198504, Russia \\ ${ }^{c}$ Department of Chemistry and Biochemistry, Swarthmore College, Swarthmore, \\ Pennsylvania 19081, USA
}

Received 20th February 2004, Accepted 21st April 2004

First published as an Advance Article on the web 18th May 2004

\begin{abstract}
The ion-pair states of molecular iodine provide a unique system for studying the efficiency, selectivity, and mechanisms of collision-induced non-adiabatic transitions. Non-adiabatic transitions between the first-tier ion-pair states in collisions with molecular partners and rare gases are analyzed and discussed. The qualitative features of the rate constants and product state distributions under single collision conditions are summarized and interpreted in terms of appropriate theoretical approaches. Two mechanisms for the non-adiabatic transitions are clearly identified. The first, operative for collisions involving molecular partners possessing permanent or transition electrostatic moments, is highly selective. It connects the initially prepared level in the $\mathrm{E} 0_{\mathrm{g}}^{+}$electronic state with the near-resonant vibronic level of the $\mathrm{D} 0_{\mathrm{u}}^{+}$state with a minimum change of the total angular momentum. In an extreme quasi-resonant case when the gap between initial and final rovibronic level is less than $1 \mathrm{~cm}^{-1}$, this mechanism has a giant cross section, 40 times that of a gas kinetic collision. An electrostatic model, which includes the coupling of the giant E-D transition dipole moment with a moment of the colliding partner and the semiclassical Born approximation, provides a plausible interpretation of this mechanism. A second mechanism is shown to govern collisions with rare gas atoms. It results in population of several ion-pair states and broad distributions over rovibronic levels. This mechanism is successfully interpreted by quantum scattering calculations based on the diatomics-in-molecule diabatic potential energy surfaces and coupling matrix elements. The calculations provide good agreement with experimental measurements and reveal different mechanisms for the population of different electronic states. Unexplained features of the non-adiabatic dynamics and directions of future work are outlined.
\end{abstract}

\section{Introduction}

Collision-induced non-adiabatic transitions (CINAT) in the gas phase play a fundamental role in the kinetics and dynamics of highly excited molecular electronic states. They are responsible for radiative emission and energy transfer in the atmosphere, energy pooling and conversion in laser media, relaxation phenomena in chemiluminescence processes, plasma formation, and in many other situations where electronically-excited states are involved. ${ }^{1-5}$ An understanding of collision-induced non-adiabatic dynamics is also essential for interpretation and modeling of various photoinitiated processes in clusters, liquids, and solids, where the role of intermolecular interaction is greatly magnified through the formation of solvation shell(s) and multiple collisions between a molecule and a solvent.

A variety of collision-induced processes, which manifest themselves through line broadening, sensitization and quenching of fluorescence, pressure dependence, etc., have been discovered in gas-phase electronic spectroscopic studies. In most cases, however, such studies only indicate the existence of non-adiabatic relaxation pathways, or at most roughly estimate their efficiency. Early works concerning CINAT suffered from a lack of resolution which prevented selective population of an initial energy level. Improvements in laser techniques have made it possible to resolve CINAT between selected vibronic levels (see, e.g., refs. 6-8 and references therein). Particularly noteworthy are studies in which both the initial and final states of the molecule are monitored with a resolution sufficient to control and interrogate individual rotational and fine structure levels (see, e.g., refs. 9-11 and references therein). These studies are limited in number and mainly focused on light diatomic systems with sparse energy levels such as $\mathrm{CO}^{+}, \mathrm{CN}, \mathrm{NO}, \mathrm{N}_{2}, \mathrm{~N}_{2}{ }^{+}$, and $\mathrm{SiCl}$. As reviewed by Dagdigian, ${ }^{10}$ the results for different systems and transitions reveal distinct propensity rules for rotational and vibrational energy transfer that accompanies CINAT. Many features of these processes are still challenging to interpret. Complete understanding of CINAT dynamics and insight into CINAT mechanisms requires further detailed, systematic, and stateof-the-art experimental and theoretical investigation.

A particularly fruitful line of attack will be studies of test systems in which a variety of non-adiabatic processes can occur. Investigating the dependence of CINAT efficiencies and branching ratios on collision partner, electronic, vibrational, and rotational excitation, collision energy, etc., will make it possible to gain a wealth of information helpful for establishing general trends and useful models applicable for 
much wider classes of systems and processes. In the present article we demonstrate that CINAT between the ion-pair (IP) states of the diatomic iodine molecule is a good candidate for such detailed and comprehensive studies.

In the homonuclear diatomic halogens, the IP states lie just above the manifold of valence states and correlate to the ionized $\mathrm{I}^{+}\left({ }^{3} \mathrm{P}_{j},{ }^{1} \mathrm{D}_{2},{ }^{1} \mathrm{~S}_{0}\right)+\mathrm{I}^{-}\left({ }^{1} \mathrm{~S}_{0}\right)$ dissociation limits. ${ }^{4,12-14}$ (In contrast, the valence states arise from interaction of two neutral $\mathrm{I}\left({ }^{2} \mathrm{P}_{j}\right)$ atoms.) The system of IP states exhibits the following features.

1. IP states of distinct symmetries form multiple tiers consisting of a set of bound potential energy curves (PECs) embedded into each other. The first tier correlates to $\mathrm{I}^{+}\left({ }^{3} \mathrm{P}_{2}\right)+$ $\mathrm{I}^{-}\left({ }^{1} \mathrm{~S}_{0}\right)$ and consists of six states, namely, $\mathrm{D}^{\prime} 2_{\mathrm{g}}, \beta 1_{\mathrm{g}}, \mathrm{D} 0_{\mathrm{u}}^{+}$, $\mathrm{E} 0_{\mathrm{g}}^{+}, \gamma 1_{\mathrm{u}}$, and $\delta 2_{\mathrm{u}}$, in order of increasing $T_{\mathrm{e}}$. The symmetry is specified within the Hund case (c) classification $\Omega_{w}^{\sigma}$, where $\Omega$ is the projection of the total electronic angular momentum onto the internuclear axis, $w=\mathrm{u}, \mathrm{g}$ specifies the parity with respect to the permutation of identical nuclei, and $\sigma$ specifies the parity of $\Omega=0$ states with respect to reflection in the vertical plane. The best available spectroscopic PECs of these states are shown in Fig. 1.

2. The equilibrium distances and spectroscopic constants of the IP states within each tier are similar, but not exactly equal. As a result, the patterns of rovibrational levels within each electronic state are irregularly shifted with respect to other electronic states of the same tier and may exhibit accidental resonances. Likewise, the Franck-Condon (FC) factors between the vibrational levels of two electronic states are irregularly distributed. The spectroscopic constants of the first and second tier IP states are well known (see, e.g., refs. 4,13,15 and references therein).

3. The emission and absorption spectroscopy of IP states is dominated by parallel transitions to predominantly a single valence electronic state with the same $\Omega$ quantum number. The IP state radiative lifetimes are quite short $\left(4.5-38 \mathrm{~ns}^{16}\right)$ allowing one to study CINAT dynamics under single-collision conditions with pressures as high as 1 Torr. Finally, a single rovibronic level in an IP state can be populated by means of the optical-optical double resonance (OODR) method.

The above features indicate that $\mathrm{I}_{2}$, when excited to an IP state, should exhibit very rich non-adiabatic collision-induced

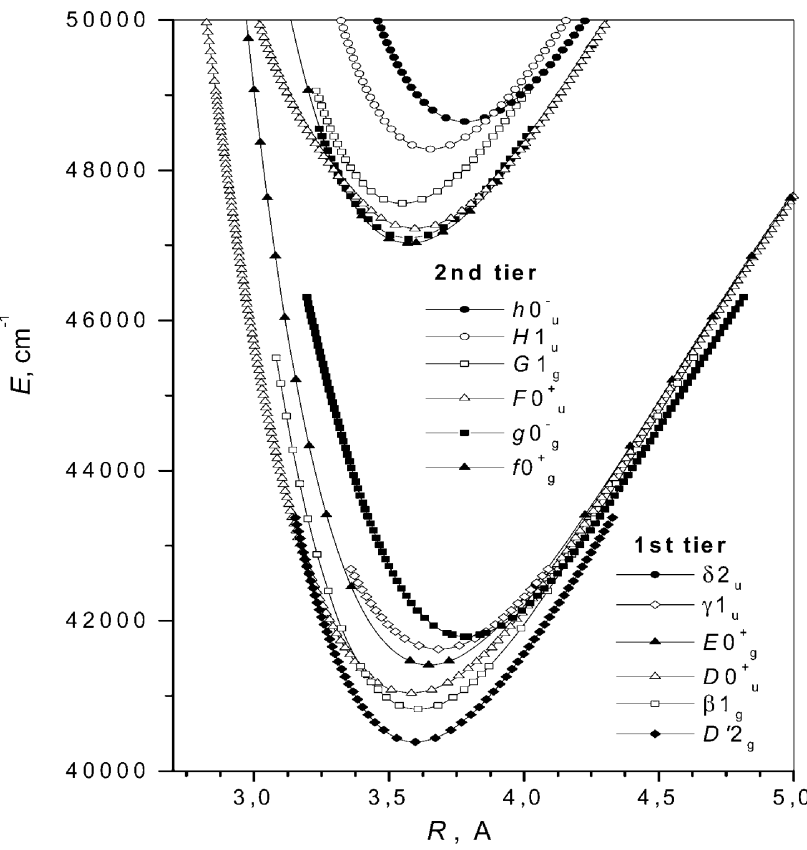

Fig. 1 Best available spectroscopic potentials for the first- and second-tier IP states of $I_{2}$ molecule. See Section 2 for references to the original papers. dynamics characteristic of perturbation-irrelevant CINAT. ${ }^{10,17}$ A careful study of this phenomenon can shed the light on the following questions.

(i) If one of the IP states is selectively excited, what final states will be populated by CINAT? What selection or propensity rules govern the non-adiabatic transitions among the dense manifold of IP states and how they are related to the symmetry of the states ( $\Omega, w$ and $\sigma$ values as well as the asymptotic $j$ value of the $\mathrm{I}^{+}$cation)?

(ii) How do the efficiencies of the non-adiabatic transitions depend on the initial and final vibrational states? For example, are near-resonant final vibrational states favored according to the energy gap law, or are those with large FC overlaps preferentially populated as prescribed by the Franck-Condon principle?

(iii) What propensity rules hold for the rotational quantum number changes in the processes under study? How does the efficiency of CINAT depend on the degree of rotational excitation?

(iv) How do all these degrees of freedom-electronic, vibrational, and rotational - interplay with one another? For example, do accidental vibrational resonances and/or extreme variations in FC overlaps alter the electronic branching ratios?

(v) Is there a significant dependence of the specificity of the CINAT on the chemical identity of the collision partner, in particular, the dipole and quadrupole moments and polarizabilities?

(vi) What model mechanism(s) can be used to describe this type of CINAT?

These questions, important for understanding any non-adiabatic event involving a diatomic molecule with a relatively high density of electronic states, can be answered by means of fully state-resolved laser spectroscopic techniques implemented under single-collision conditions and supplemented by accurate theoretical calculations. Collision-induced energy transfer between the IP states of the iodine molecule is of practical interest as well. For example, CINAT is responsible for the $\mathrm{D}^{\prime} 2_{\mathrm{g}} \rightarrow \mathrm{A}^{\prime} 2_{\mathrm{u}}$ lasing action. ${ }^{18-20}$ Transitions to and among IP states are widely explored in studies of iodine photodynamics in inert environments (compressed gases, clusters, liquids, matrices, etc.): a paradigm for understanding solvation effects on molecular processes. ${ }^{21-30}$

The history of studies of CINAT in the iodine IP states reflects the progress in molecular spectroscopy in the past century. The first observation of the non-adiabatic transitions between the IP states of the iodine molecule dates back to $1924,{ }^{31}$ only thirteen years after the first observation of nonadiabatic quenching of the valence $\mathrm{B} 0_{\mathrm{u}}^{+}$state. ${ }^{32,33}$ It was later found that excitation of the ground $\mathrm{X} 0_{\mathrm{g}}^{+}$state to the $\mathrm{D} 0_{\mathrm{u}}^{+}$ first-tier IP state results in efficient $\mathrm{D}^{\prime} 2_{\mathrm{g}} \rightarrow A^{\prime} 2_{\mathrm{u}}$ fluorescence through collision-induced relaxation to the $\mathrm{D}^{\prime}$ state. This system attracted many researchers, ${ }^{34}{ }^{47}$ mainly due to observation of lasing action on the $\mathrm{D}^{\prime} \rightarrow \mathrm{A}^{\prime}$ transition. ${ }^{18-20,48-54}$ Because of the $\mathrm{D} \leftarrow \mathrm{X}$ excitation sources utilized-electric discharges, lamps with monochromators, ArF lasers - it was impossible to achieve single vibrational level resolution in the excitation of the $\mathrm{D}$ state. The measurements were performed under multiple-collision conditions and the kinetic analysis utilized a onelevel approximation. Therefore, the rate constants reported in these investigations (and summarized in ref. 55) represent only averaged effective rate parameters. These early studies clearly demonstrated, however, the high efficiency of CINAT between the IP states. Tellinghuisen and co-workers showed that the IP state population after discharge excitation rapidly evolves to an almost thermal distribution ${ }^{40}$ and that $\mathrm{D}^{\prime} \rightarrow \mathrm{A}^{\prime}$ fluorescence results from $\mathrm{D} \leftarrow \mathrm{X}$ excitation even at very low collision energies in a low pressure free-jet expansion in $\mathrm{Ar}^{56}$

Application of the OODR technique have made possible truly state-resolved investigations of IP state dynamics. ${ }^{57}$ Koffend et al. ${ }^{58}$ observed $\mathrm{D}^{\prime} \rightarrow \mathrm{A}^{\prime}$ and $\mathrm{D} \rightarrow \mathrm{X}$ emissions 
following selective excitation of $\mathrm{E} 0_{\mathrm{g}}^{+}$state. Min et al. $^{59}$ reported the population of a single rovibrational level of a "new IP state $\mathrm{E}_{2}$ " (either the $\mathrm{E} 0_{\mathrm{g}}^{+}$state or a $\Omega$-doublet component of the $\beta 1_{\mathrm{g}}$ state $^{60}$ ) and its effective depopulation rate constant by nitrogen at rather high pressures. According to refs. 16 and 61, state-selective two-photon excitation produces emission from almost all of the IP states of the first tier, similar to discharge excitation. ${ }^{40}$ However, single-collision conditions were not fulfilled in these measurements. The first studies of CINAT at single-collision conditions have been presented in ref. 62. In this and subsequent works ${ }^{63,64}$ the $\mathrm{D} \rightarrow \mathrm{X}$ fluorescence resulting from $\mathrm{E} \leftarrow \mathrm{B} \leftarrow \mathrm{X}$ OODR excitation was resolved and the $\mathrm{D}$ state vibrational state distributions were obtained for collisions with ground-state iodine molecules and some foreign gases.

Quantitative state-resolved measurements of the absolute room temperature rate constants and product state distributions for CINAT originating in the $\mathrm{E} 0_{\mathrm{g}}^{+}$state have been initiated recently by research groups at St. Petersburg State University $^{65-69}$ and at Swarthmore College. ${ }^{70-72}$ At the same time, a framework for a theoretical description has been developed at Moscow State University. ${ }^{73-75}$ The main goal of this article is to bring together the results and experience of these active groups, in order to establish a common interpretation, to document existing inconsistencies, and to highlight promising directions for the future. While we present here data from previously published as well as recent unpublished investigations, this article merely summarizes an extensive body of detailed results, and concludes with more questions than answers. We have attempted to identify the most persistent qualitative features in the experimental data and to support these generalizations with theoretical calculations, with the goal of determining model CINAT mechanisms.

In the next section we briefly introduce the experimental methods employed and basic consideration of the electronic structure of the IP states underlying the theoretical approaches. Section 3 describes experimental results and their theoretical interpretation. In Section 4, we discuss general features of the CINAT mechanisms, remaining questions, and identify areas of future exploration.

\section{Experimental and theoretical background}

\subsection{Experimental technique}

The experimental techniques used at Swarthmore and St. Petersburg to investigate the CINAT dynamics of $\mathrm{I}_{2}(\mathrm{E})$ are similar (see details in refs. 65-72,76,77). Single rovibrational levels of the $\mathrm{E} 0_{\mathrm{g}}^{+}$state were populated through an OODR excitation scheme:

$$
\mathrm{E}\left(0_{\mathrm{g}}^{+}, v_{\mathrm{E}}, J_{\mathrm{E}}\right) \stackrel{\lambda_{2}}{\longleftarrow} \mathrm{B}\left(0_{\mathrm{u}}^{+}, v_{\mathrm{B}}, J_{\mathrm{B}}\right) \stackrel{\lambda_{1}}{\longleftarrow} \mathrm{X}\left(0_{\mathrm{g}}^{+}, v_{\mathrm{X}}, J_{\mathrm{X}}\right),
$$

where $v_{\mathrm{E}}$ is the vibrational quantum number and $J_{\mathrm{E}}$ is the total angular momentum of the molecule in the $\mathrm{E}$ electronic state. The initial $v_{\mathrm{E}}=0, J_{\mathrm{E}}=55,98$ and $v_{\mathrm{E}}=1,2, J=55$ levels studied at Swarthmore were populated through $\mathrm{B} \leftarrow \mathrm{X}$ excitation to one of the following B state levels: $v_{\mathrm{B}}=20, J_{\mathrm{B}}=56$ $\left(\lambda_{1}=559.95 \mathrm{~nm}\right), v_{\mathrm{B}}=21, J_{\mathrm{B}}=97\left(\lambda_{1}=559.96 \mathrm{~nm}\right), v_{\mathrm{B}}=$ $21, J_{\mathrm{B}}=56\left(\lambda_{1}=557.18 \mathrm{~nm}\right)$ or $v_{\mathrm{B}}=23, J_{\mathrm{B}}=56\left(\lambda_{1}=\right.$ $551.90 \mathrm{~nm})$. The $v_{\mathrm{E}}=8-55, J_{\mathrm{E}}=51 / 53,56 / 58, \approx 80, \approx 110$ levels investigated at St. Petersburg were accessed through the $v_{\mathrm{B}}=32, J_{\mathrm{B}}=52,57 ; v_{\mathrm{B}}=33, J_{\mathrm{B}}=82,86 ; v_{\mathrm{B}}=34$, $J_{\mathrm{B}}=102,107\left(\lambda_{1}=532.24 \mathrm{~nm}\right)$ levels.

Emission from the IP states was collected with a f/1.2 fused silica lens (Swarthmore) or f/2.5 fused silica condenser (St. Petersburg) and projected onto the entrance slit of a monochromator equipped with a PMT detector (St. Petersburg) or CCD camera (Swarthmore). The spectral dependence of the sensitivity of the condensor/monochromator/PMT system utilized in St. Petersburg was determined at a geometry identical to that used for spectral measurements within an accuracy that ranges from $\pm 5 \%$ at $450 \mathrm{~nm}$ to $\pm 15 \%$ at $220 \mathrm{~nm}$. Typical spectral resolutions were $0.16 \mathrm{~nm}$ (Swarthmore), and 0.2-0.3 nm (St. Petersburg).

The samples consisted of iodine at pressures that varied from 20 to 160 mTorr (Swarthmore) or from 70 to $300 \mathrm{mTorr}$ (St. Petersburg), along with a variable pressure of a foreign gas collision partner. All pressures were measured with a precision of \pm 1 mTorr (Swarthmore) or \pm 20 mTorr (St. Petersburg).

The distributions of rovibrational populations for the electronic states populated by CINAT were determined by simulation of the emission spectra. The simulations were performed using FC factors generated by the LEVEL program ${ }^{78}$ (Swarthmore) or the original codes COOLA and SIMUL (St. Petersburg). $I_{2}$ spectroscopic data and RKR curves were taken from a variety of literature sources. ${ }^{79-92}$ Due to the lack of rotational resolution, the simulations characterized only the maximum and the width of the rotational distribution. The accuracy of these parameters varies with relative population and depends on the collision partner. State-specific CINAT rate constants were determined from the pressure dependence of the integrated $\mathrm{E} \rightarrow \mathrm{A} 1_{\mathrm{u}}, \mathrm{B} 0_{\mathrm{u}}^{+}, \mathrm{B}^{\prime \prime} 1_{\mathrm{u}} ; \mathrm{D} \rightarrow \mathrm{X} ; \mathrm{D}^{\prime} \rightarrow \mathrm{A}^{\prime}$ $2_{\mathrm{u}}$, and $\beta \rightarrow \mathrm{A} 1_{\mathrm{u}}$ emission intensities taking into account both radiative and collisional population/depopulation processes. Several weaker bands assigned to emission from the $\delta$ state were detected, though not analyzed in detail. All the measurements were performed under approximately single-collision conditions. It was found that the relaxation processes within a single electronic state can be neglected in the analysis.

\subsection{Electronic structure of IP states}

The electronic structure of IP states of an isolated halogen molecule can be considered within two distinct frameworks. ${ }^{93}$ The conventional molecular orbital-electronic configuration picture was explored in the classic paper by Mulliken ${ }^{94}$ and is naturally appropriate in the context of ab initio calculations. ${ }^{95,96}$ The second approach, more conveniently used in semiempirical studies, and known as the "pure precession",93 or "atoms-in-molecule" model, ${ }^{97}$ originates from the considerations of Van Vleck and Herzberg.

In this article, we will use a version of the latter approach, whose application to halogen IP states is described in most detail by Jewsbury and Lawley. ${ }^{93}$ In brief, the pure precession model expresses the molecular electronic wave function using the many-electron functions of isolated atoms. The Hund case (c) wave function of the $\mathrm{AB}$ molecule in the state $n \Omega_{w}^{\sigma}$ is represented as a linear combination of the products of spin-orbit coupled wave functions of atoms A and B:

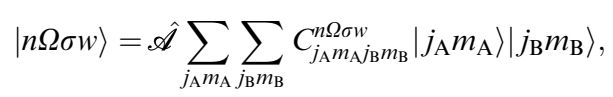

where $\hat{\mathscr{A}}$ is the partial antisymmetrization operator, $n$ identifies the molecular state (e.g., by spectroscopic lettering notations), and $\left|j_{\alpha} m_{\alpha}\right\rangle$ is the electronic wave function of atom $\alpha$ $(=\mathrm{A}$ or $\mathrm{B})$ parameterized by the total electronic angular momentum $j_{\alpha}$ and its projection $m_{\alpha}$ onto the internuclear axis. The coefficients $C$ in eqn. (1) depend on internuclear distance $r$.

In the limit of separated atoms, $r \rightarrow \infty$, eqn. (1) is greatly simplified because the coefficients $C$ are fixed by symmetry requirements. In the particular case of the IP states of the halogen molecule $\mathrm{X}_{2}$ correlating to the $\mathrm{X}^{+}\left({ }^{3} \mathrm{P}_{j}\right)+\mathrm{X}^{-}\left({ }^{1} \mathrm{~S}_{0}\right)$ limit, one has

$$
|n \Omega \sigma w ; j\rangle=\frac{1}{\sqrt{2}}\left[|j m\rangle|00\rangle+(-1)^{p}|00\rangle|j m\rangle\right],
$$

where $m=\Omega$ and $p$ is determined by the parity $w$ (and $\sigma$, if $\Omega=0$ ). 
Eqn. (2) provides a convenient basis for constructing more accurate expressions for the wave functions (1). It can also be directly used for describing IP states if, to the zero-order approximation, one neglects the configuration interaction mixing between different IP states. (Alternatively, one can consider the function given by eqn. (2) as the diabatic electronic wave function of the IP state and neglect the diabatic coupling with other states.) If one neglects in addition the configuration interaction effects in the atomic structure (pertinent to $\mathrm{X}^{+}$ cations), ${ }^{93}$ one can consider $|j m\rangle$ functions within the standard $L S$ (Russell-Saunders) scheme. In this case, according to eqn. (2), $j$ is a good quantum number and there is no mixing between IP states of different tiers. It has been argued ${ }^{98}$ that the similarity and small splitting of the IP potential energy curves indicate the validity of this simple model for distances close to the equilibrium bond length, $r_{\mathrm{e}}$. To avoid confusion with the more exact pure precession model, we will refer to approximation (2) as the "asymptotic model".

Within the asymptotic model, each IP state is characterized by $n, \Omega, w, \sigma$, and $j$ quantum numbers. The asymptotic wave functions (2) of the first-tier IP states are listed in Table 1 (note that in the wave functions given in refs. 73 and 99 the signs of gerade and ungerade combinations have been mistakenly interchanged; due to the symmetry of the problem this does not lead to errors in the subsequent formulas). For degenerate $(\Omega \neq 0)$ states the representation with signed projections is used for simplicity; the wave functions of definite parity which provide a more convenient basis for dynamical calculations can be easily constructed by taking plus and minus linear combinations of the wave functions with opposite projections. ${ }^{75}$

\section{CINAT dynamics}

\subsection{Collisions with iodine, nitrogen, and $\mathrm{CF}_{4}$ molecules}

3.1.1 Experimental observations. $\mathrm{E} \rightarrow \mathrm{D}$ CINAT was observed at Swarthmore when $\mathrm{I}_{2}\left(\mathrm{E}, v_{\mathrm{E}}=0\right)$ collides with $\mathrm{I}_{2}(\mathrm{X}){ }^{70}$ The rate constant (see Fig. 2$)$ is equal to $(4.0 \pm 0.7) \times$ $10^{-11}$ (throughout this article, all rate constant values are given in $\mathrm{cm}^{3}$ molecule ${ }^{-1} \mathrm{sec}^{-1}$ ) and corresponds to a hard-sphere cross section value of $20 \AA^{2}$, i.e. 4-5 times smaller than a gas-kinetic cross section. Identical cross sections and rate constants are found for excitation of $J_{\mathrm{E}}=55$ and 98 . The vibrational product state distribution is depicted in Fig. 3. It peaks at the $v_{\mathrm{D}}=1,2$ levels, whereas the initial E, $v_{\mathrm{E}}=0$ level is nearly resonant with the $v_{\mathrm{D}}=4$ level in the $D$ electronic state. The relative lack of population in the latter demonstrates that a propensity to populate levels with the smallest energy gaps is not valid. Alternatively, the E, $v_{\mathrm{E}}=0-\mathrm{D}$ Franck-Condon factors peak at $v_{\mathrm{D}}=0$, and decline rapidly as $v_{\mathrm{D}}$ increases. Clearly, there is no overwhelming propensity directing population only to those levels with large vibrational overlap with the initial state. The overall shape of the distributions suggests a balance between these two effects, though one in which more weight is given to FC factors. Use of the combined FC-energy gap model by Katayama et al. ${ }^{100}$ confirms that both effects are important, but it cannot be determined whether this model

Table 1 Wave functions of the first-tier states of halogen molecules within the asymptotic model

\begin{tabular}{ll}
\hline State & Asymptotic wave function \\
\hline $\mathrm{D}^{\prime} 2_{\mathrm{g}}(j=2)$ & {$[|2 \pm 2\rangle|00\rangle+|00\rangle|2 \pm 2\rangle] / \sqrt{ } 2$} \\
$\beta 1_{\mathrm{g}}(j=2)$ & {$[|2 \pm 1\rangle|00\rangle+|00\rangle|2 \pm 1\rangle] / \sqrt{ } 2$} \\
$\mathrm{D} 0_{\mathrm{u}}^{+}(j=2)$ & {$[|20\rangle|00\rangle-|00\rangle|20\rangle] / \sqrt{ } 2$} \\
$\mathrm{E} 0_{\mathrm{g}}^{+}(j=2)$ & {$[|20\rangle|00\rangle+|00\rangle|20\rangle] / \sqrt{ } 2$} \\
$\gamma 1_{\mathrm{u}}(j=2)$ & {$[|2 \pm 1\rangle|00\rangle-|00\rangle|2 \pm 1\rangle] / \sqrt{ } 2$} \\
$\delta 2_{\mathrm{u}}(j=2)$ & {$[|2 \pm 2\rangle|00\rangle-|00\rangle|2 \pm 2\rangle] / \sqrt{ } 2$} \\
\hline
\end{tabular}

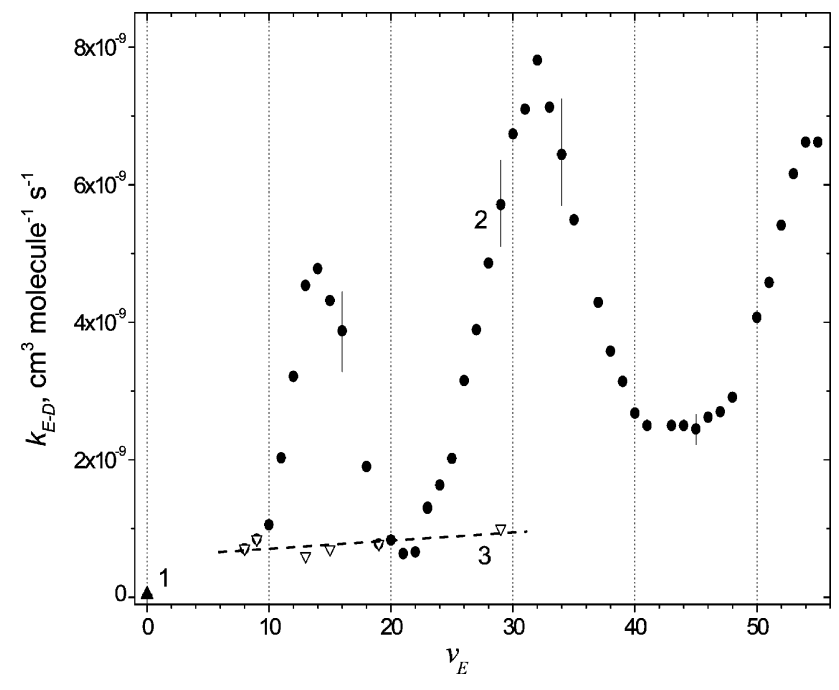

Fig. 2 Total rate constants for $\mathrm{E}, v_{\mathrm{E}} \rightarrow \mathrm{D}$ transitions in $\mathrm{I}_{2}(\mathrm{E})+\mathrm{I}_{2}(\mathrm{X})$ collisions. The results from the Swarthmore (1) and St. Petersburg $(2,3)$ groups are presented. Data set 3 represents the rate constants summed over the transitions to all final $\mathrm{D}, v_{\mathrm{D}}$ levels except the nearresonant one.

incorporates the correct balance between FC and energy gap effects.

CINAT with collision partners $\mathrm{M}=\mathrm{I}_{2}, \mathrm{~N}_{2}, \mathrm{CF}_{4}$ originating in selected rovibrational E-state levels $v_{\mathrm{E}}=8-55, J_{\mathrm{E}} \approx$ 55,85 , and 105 were investigated at St. Petersburg. For $\mathrm{I}_{2}(\mathrm{E})+\mathrm{I}_{2}(\mathrm{X})$ collisions, the iodine pressure ranged from 0.07 to 0.3 Torr. When $\mathrm{N}_{2}$ or $\mathrm{CF}_{4}$ was the collision partner, the iodine pressure was fixed at $\approx 0.2$ Torr and a variable pressure of the foreign gas was added. The ratios of $\mathrm{D} \rightarrow \mathrm{X}$ to $\mathrm{E} \rightarrow \mathrm{B}$ emission intensities, total rate constants and vibrational/rotational product state distributions for $\mathrm{E} \rightarrow \mathrm{D}$ CINAT were determined. ${ }^{65-68}$

$M=I_{2}$. As in the case of $v_{\mathrm{E}}=0$ excitation, CINAT was observed only to D $0_{u}^{+}$state. ${ }^{65,67,76}$ As shown in Fig. 2, the total rate constant for the $\mathrm{E} \rightarrow \mathrm{D}$ transition varies between 7 and $80 \times 10^{-10}$, much higher than the upper bound of $10^{-10}$ estimated by Teule et al. ${ }^{64}$ The rate constant also exhibits sharp maxima as a function of $v_{\mathrm{E}}$. The hard-sphere collision cross section at the maxima reaches the huge value of 3300 $\AA^{2}$, approximately forty times larger than either the gas-kinetic cross section or the cross sections of other perturbationirrelevant CINAT. ${ }^{10}$ These transitions have been labelled

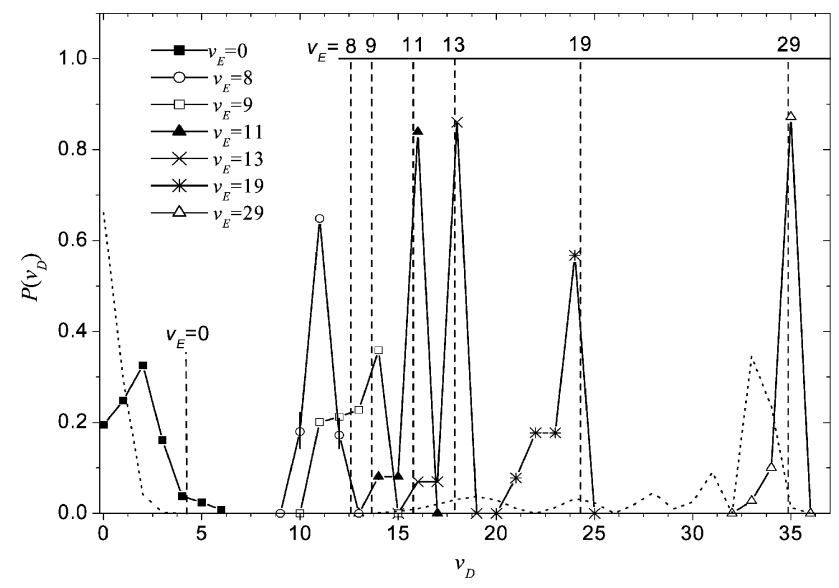

Fig. 3 Vibrational product state distributions for $\mathrm{E}, v_{\mathrm{E}} \rightarrow \mathrm{D}, v_{\mathrm{D}}$ transitions in $\mathrm{I}_{2}(E)+\mathrm{I}_{2}(\mathrm{X})$ collisions measured by Swarthmore $\left(v_{\mathrm{E}}=0\right)$ and St. Petersburg groups. The positions of the initial levels are marked by vertical dashed lines. Dotted lines represent the FC factor distributions for $v_{\mathrm{E}}=0$ and 29. 
"approach-induced" to emphasize their extremely long-range character. ${ }^{65}$ The positions of the rate constant maxima strictly correspond to the quasi-resonant cases when there exists a final $v_{\mathrm{D}}$ level whose energy gap to initial $v_{\mathrm{E}}$ level does not exceed 25 $\mathrm{cm}^{-1}$. Larger energy gaps between the initial and the closest final vibrational level results in progressively smaller rate constants. No dependence of the total or the vibrationallyresolved rate constants on initial rotational angular momentum, $J_{\mathrm{E}}$, is found.

The D state vibrational level distributions indicate that the propensity for population of levels with the smallest energy gaps exists even if the quasi-resonance condition is not fulfilled. As seen in Fig. 3, the distributions are very narrow with sharp maxima at the near-resonant final levels, except for $v_{\mathrm{E}}=8$, in good agreement with the previous measurements. ${ }^{64}$ Practically no correlation with FC factors is observed. If one removes the contribution of the near-resonant transition from the total rate constant, the remaining non-resonant component is only weakly dependent on $v_{\mathrm{E}}$ (see curve 3 on Fig. 2) and corresponds to a hard-sphere cross section of order $300 \AA^{2}$, still significantly larger than the gas-kinetic cross section. When extrapolated to $v_{\mathrm{E}}=0$, this function gives a value exceeding the measured one $\mathrm{e}^{70}$ by an order of magnitude.

Rotational product state distributions for the quasi-resonant transitions with $|\Delta E| \leq 25 \mathrm{~cm}^{-1}$ (rate constants $c a .8 \times 10^{-9}$ ) follow a "low- $\Delta J$ " propensity rule, i.e., $\Delta J=J_{\mathrm{E}}-J_{\mathrm{D}}= \pm 1$ transitions barely dominate. The rotational distributions for transitions with larger energy gaps (and therefore smaller rate constants) were hard to establish quantitatively. Spectral simulations provided an indication that all the transitions with rate constants less than $10^{-9}$ likely follow the same low$\Delta J$ propensity rule ${ }^{67}$ in accord with the other data. ${ }^{63,64,70}$ $M=N_{2}$. The D state populations resulting from collisions with $\mathrm{N}_{2}$ are quite similar to those observed following collisions with rare gas atoms (see below). The rate constant exhibits larger variations with $v_{\mathrm{E}}$ (see Fig. 4) and corresponds to hardsphere cross sections approximately 1-3 times gas kinetic. As shown in Fig. 5, the vibrational product state distributions have sharp maxima at the levels lying $50-120 \mathrm{~cm}^{-1}$ below resonance and broad tails. The rotational distributions obey a "high- $\Delta J$ " propensity rule, and have an approximately Gaussian shape centered at $\Delta J= \pm 1$ with a half-width at halfmaximum $\Delta J= \pm(7-9)$ for $v_{\mathrm{E}}=8$ (see also refs. 64 and 68). $M=C F_{4}$. The $\mathrm{D}$ state vibrational distributions resulting from collisions with $\mathrm{CF}_{4}$ can be classified into one of four groups according to the size of energy gaps to the mostly populated D-state vibrational level (see Fig. 6) ${ }^{66,68}$ The groups I, II, III and IV correspond to transitions with energy gaps of $\leq 400$,

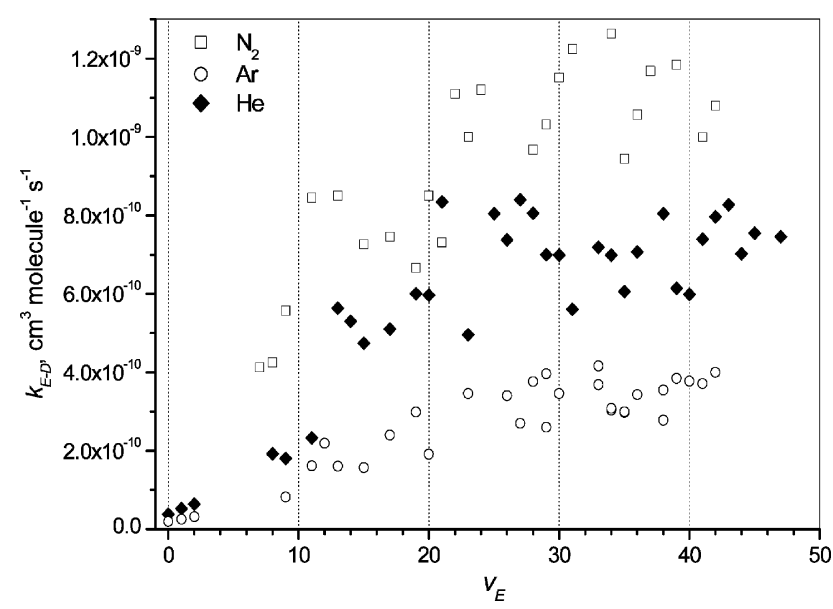

Fig. 4 Total rate constants for $\mathrm{E}, v_{\mathrm{E}} \rightarrow \mathrm{D}$ transitions in $\mathrm{I}_{2}(\mathrm{E})+\mathrm{N}_{2}$, $\mathrm{Ar}$, and He collisions. Results for $\mathrm{He}$ and $\mathrm{Ar}$ at $v_{\mathrm{E}} \leq 2$ by Swarthmore group, other results by St. Petersburg group.
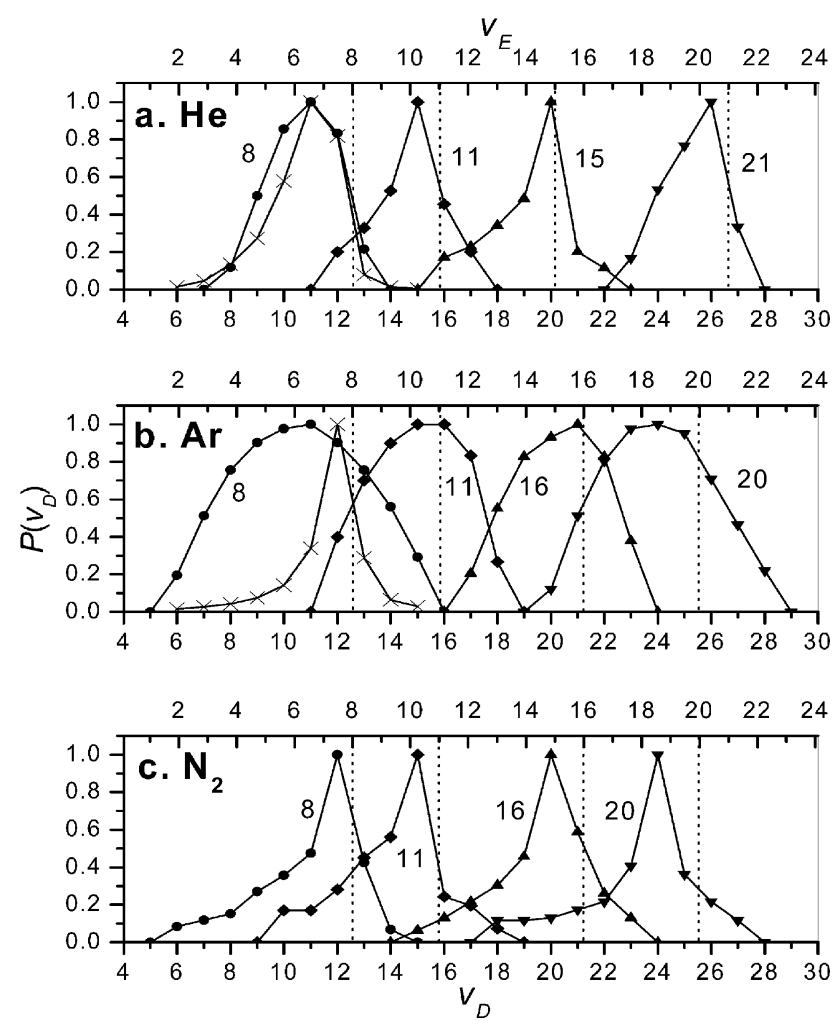

Fig. 5 Vibrational product state distributions (normalized to unity at the maximum) for $\mathrm{E}, v_{\mathrm{E}} \rightarrow \mathrm{D}, v_{\mathrm{D}}$ transitions in $\mathrm{I}_{2}(\mathrm{E})+\mathrm{He}(\mathrm{a}), \mathrm{Ar}(\mathrm{b})$ and $\mathrm{N}_{2}$ (c) collisions. Top axes show $v_{\mathrm{E}}$ quantum numbers, vertical dotted lines indicate initial excitations $v_{\mathrm{E}}=8,11,15$ or 16 , and 20 or 21. For $\mathrm{He}$ and $\mathrm{Ar}$ at $v_{\mathrm{E}}=8$, theoretical results (EVCC MHS) are shown by crosses.

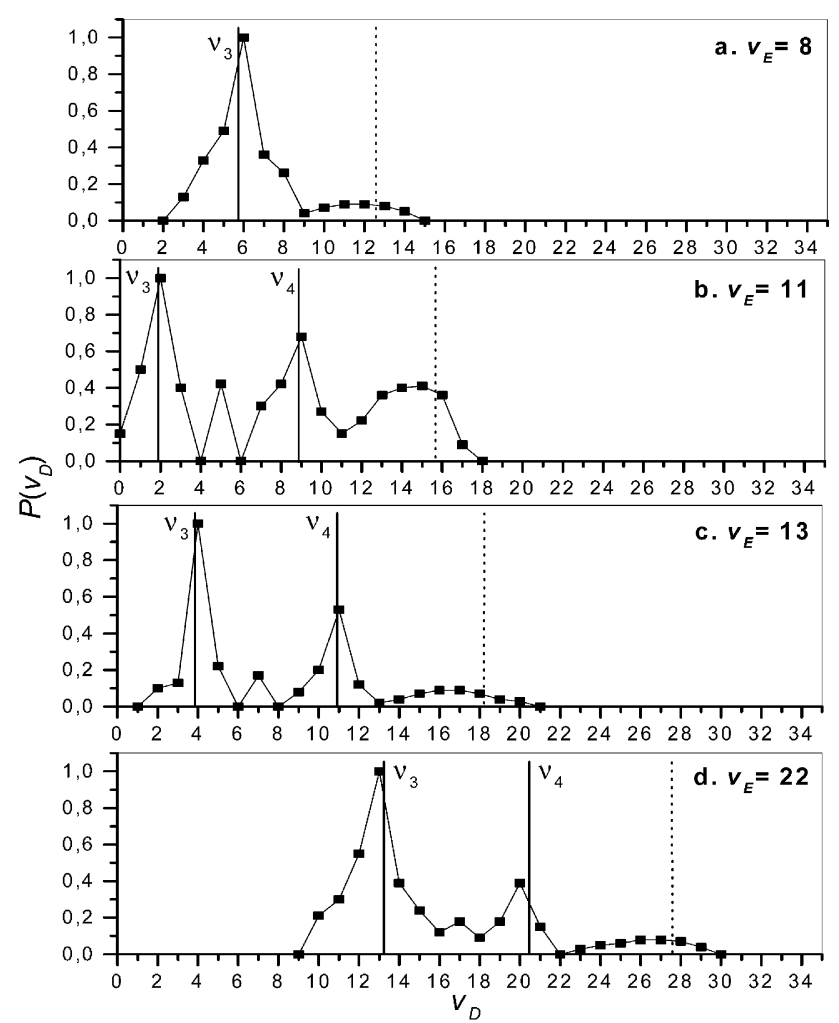

Fig. 6 Vibrational product state distributions (normalized to unity at the maximum) for $\mathrm{E}, v_{\mathrm{E}} \rightarrow \mathrm{D}, v_{\mathrm{D}}$ transitions in $\mathrm{I}_{2}(\mathrm{E})+\mathrm{CF}_{4}$ collisions at $v_{\mathrm{E}}=8(\mathrm{a}), 11$ (b), 13 (c), and 22 (d). Vertical dotted lines indicate the positions of initial level, solid lines-excitation energies of $\nu_{3}$ and $\nu_{4}$ modes of $\mathrm{CF}_{4}$ molecule. 
$\approx 630, \approx 1060$, and $\approx 1280 \mathrm{~cm}^{-1}$, respectively. Groups II and IV are assigned to transitions in which the $\mathrm{CF}_{4}$ collision partner achieves vibrational excitation to the $\nu_{4}=631.2$ and $\nu_{3}=1283 \mathrm{~cm}^{-1}$ levels, respectively. Group I involves the transitions which are not accompanied by vibrational excitation of collision partner, while group III can be attributed to $\nu_{2}+\nu_{4}$ excitation with an energy of $1066 \mathrm{~cm}^{-1}$.

Group I CINAT is similar to that observed for $\mathrm{M}=\mathrm{N}_{2}$ though with slightly lower partial rate constants $(0.8-1.9) \times$ $10^{-10}$ (cross sections of 0.3-0.7 times gas kinetic) and similar rotational product state distributions. The partial rate constants corresponding to groups II, III, and IV fall in the ranges $(0.4-1.7),(0.1-0.5)$, and $(0-2.5) \times 10^{-10}$, respectively. The rotational distributions for these transitions are similar to those obtained for near-resonant CINAT by $\mathrm{I}_{2}(\mathrm{X})$, i.e., they obey a low- $\Delta J$ propensity rule. The total $\mathrm{E} \rightarrow \mathrm{D}$ rate constant varies from 2.7 to $6.2 \times 10^{-10}$. A very intriguing result is obtained at $v_{\mathrm{E}} \approx 30$ : the rate constant for $J_{\mathrm{E}} \approx 110$ is $2.9 \times 10^{-9}$ which is 5-6 times larger than those for $J_{\mathrm{E}} \approx 55$, $80.66,68$

For collisions with $\mathrm{N}_{2}$ and $\mathrm{CF}_{4}$, additional emission bands were detected. As argued in Section 3.2.1 (below), one of features indicates weak collisional population of the $\delta 2_{\mathrm{u}}$ state.

3.1.2 Theoretical interpretation-electrostatic model and semiclassical born approximation. A transparent semiquantitative interpretation of CINAT with molecular collision partners relies upon the electrostatic model ${ }^{66-69}$ introduced by Lawley for the collisions of iodine in the IP states with ground state iodine. ${ }^{98}$ In this model the diabatic couplings between IP states are determined by the long-range multipole expansion. The lowest-order term in this expansion for neutral molecules is proportional to the dipole moment operator. For a homonuclear iodine molecule, the diagonal matrix elements of the dipole moment operator using electronic wave functions $|n \Omega \sigma w ; j\rangle$ in eqn. (1) or (2) obviously vanish, but the non-diagonal elements do not. Therefore, according to electrostatic model, CINAT is subjected by the same selection rules as radiative dipole transitions. Together with the constrains of the asymptotic model, these selection rules can be formulated as follows:

(1) Transitions connecting the states of different tiers of IP states $(\Delta j \neq 0)$ are forbidden;

(2) The only transitions allowed are accompanied by $\Delta \Omega=0,1$ and a change of inversion parity $w=\mathrm{u}, \mathrm{g}$;

(3) The "parallel" $\Omega_{\mathrm{g}}^{\sigma} \leftrightarrow \Omega_{\mathrm{u}}^{\sigma}$ transitions with $\Delta \Omega=0$ are dominant;

(4) The total angular momentum must change by one unit, $\Delta J= \pm 1$.

Lawley argued that the transition dipole moments for close lying pairs of $\Omega_{\mathrm{g}}^{\sigma}-\Omega_{\mathrm{u}}^{\sigma}$ states belonging to the same tier are very large and grow almost linearly with internuclear distance. ${ }^{98}$ Thus, the electrostatic model explains two important experimental findings: the predominance of $\mathrm{E} \rightarrow \mathrm{D}$ CINAT and the low- $\Delta J$ propensity for rotational energy transfer.

A more quantitative analysis of the quasi-resonant transitions is possible in the frame of the semiclassical Born approximation (see, e.g., refs. 98,101-104). Within the electrostatic model, the non-adiabatic coupling between $\mathrm{E}$ and $\mathrm{D}$ states is expressed at long distances as

$$
V\left(\boldsymbol{r}, \boldsymbol{r}_{\mathrm{M}}, \boldsymbol{R}\right)=\mu_{\mathrm{ED}}(r) \sum_{l} Q_{\mathrm{M}}^{l}\left(\boldsymbol{r}_{\mathrm{M}}\right) C_{l}\left(\hat{\boldsymbol{r}}_{,} \hat{\boldsymbol{r}}_{\mathrm{M}}, \hat{\boldsymbol{R}}\right) / R^{l+2},
$$

where $\boldsymbol{r}$ is the vector joining two iodine atoms, $\boldsymbol{r}_{\mathrm{M}}$ is the vector of all internal coordinates of the collision partner $\mathrm{M}, \boldsymbol{R}$ is the vector joining the center of mass of the $\mathrm{I}_{2}$ molecule and collision partner, $\mu_{\mathrm{ED}}(r)$ is the $\mathrm{E}-\mathrm{D}$ transition dipole moment function of the $\mathrm{I}_{2}$ molecule, $Q_{\mathrm{M}}^{l}\left(\boldsymbol{r}_{\mathrm{M}}\right)$ is the $l$ th electric moment of $\mathrm{M}$, and $C_{1}\left(\hat{\boldsymbol{r}}, \hat{\boldsymbol{r}}_{\mathrm{M}}, \hat{\boldsymbol{R}}\right)$ is the anisotropy factor depending on all orientation angles.

Describing the relative motion of the partners classically by a straight-line trajectory with impact parameter $b$ and relative velocity $g$ and applying first-order semiclassical perturbation theory, one obtains for the transition probability

$$
\begin{aligned}
P_{\mathrm{E} v_{\mathrm{E}} v_{\mathrm{i}}, \mathrm{D} v_{\mathrm{D}} v_{\mathrm{f}}}(b, g)= & \sum_{l} \frac{1}{\hbar^{2} g^{2} b^{2(l+1)}} \\
& \times\left[\mu_{\mathrm{ED}}\left\langle v_{\mathrm{E}} \mid v_{\mathrm{D}}\right\rangle\left\langle v_{\mathrm{i}}\left|Q_{\mathrm{M}}^{l}\right| v_{\mathrm{f}}\right\rangle\right]^{2} C_{l} R_{l}(\xi),
\end{aligned}
$$

where $v_{\mathrm{i}}, v_{\mathrm{f}}$ are the initial and final vibrational quantum numbers of the partner $\mathrm{M}, C_{l}$ accumulates the dependence on angular momentum quantum numbers, $R_{l}$ is the resonance function of the Massey parameter $\xi=|\Delta E| / \hbar g$, and $|\Delta E|$ is the energy difference between the initial and final states of the colliding partners. ${ }^{102,104}$ The cross section is given by

$$
\sigma_{\mathrm{E} v_{\mathrm{E}} v_{\mathrm{i}}, \mathrm{D} v_{\mathrm{D}} v_{\mathrm{f}}}(g)=\pi b_{\mathrm{c}}^{2}+\int_{b_{\mathrm{c}}}^{\infty} P_{\mathrm{E} v_{\mathrm{E}} v_{\mathrm{i}}, \mathrm{D} v_{\mathrm{D}} v_{\mathrm{f}}}(b, g) b \mathrm{~d} b,
$$

where the cutoff impact parameter $b_{\mathrm{c}}$ is introduced to account for the hard-sphere potential by setting $P_{\mathrm{E} v_{\mathrm{E}} v_{\mathrm{i}}, \mathrm{D} v_{\mathrm{D}} v_{\mathrm{f}}}\left(b_{\mathrm{c}}, g\right)=$ $1 / 2 .^{101}$

For $\mathrm{I}_{2}(\mathrm{X})$ and $\mathrm{N}_{2}$ collision partners, the leading electrostatic term contains quadrupole moments $\Theta(l=2)$. Using Lawley's estimation for $\mu_{\mathrm{ED}}(25 \mathrm{D})^{98}$ (a comparable result has also been obtained in ab initio calculations for the $\mathrm{Cl}_{2}$ molecule ${ }^{105}$ ), the quadrupole moment value for $\mathrm{I}_{2}(\mathrm{X})$ molecule $\left(\approx 10^{-25}\right.$ esu), a typical value for $\mathrm{FC}$ factors for the transitions under study (0.2), and assuming that the resonance function is $R_{12}(\xi)=$ $1 \pm 0.2$ for the states with $|\Delta E| \leq 25 \mathrm{~cm}^{-1}$ (compensation of the resonance defect due to vibrational and rotational energy transfer in the molecular partner is assumed), one arrives at cross section estimates ranging from 2000 to $4000 \AA^{2}$ depending on the value of the coefficient $C_{l}$ (or, more precisely, the change in the rotational energy of the colliding partners). This result is in complete agreement with the very large cross sections observed for the quasi-resonant transitions.

For the CINAT with $\mathrm{M}=\mathrm{N}_{2}$, the measurements do not show sharp maxima of the cross sections at quasi-resonant conditions and the theoretical estimate must be compared with the partial cross section to a near-resonant vibrational level. According to above model, the cross sections should be approximately a factor of 7 lower than those of $\mathrm{M}=\mathrm{I}_{2}$, a result of the differences in the quadrupole moments. The measured cross sections differ by approximately a factor of 10 .

For $\mathrm{M}=\mathrm{CF}_{4}$, the leading term of electrostatic expansion (3) corresponds to $l=1$, the vibrational dipole moment of $\mathrm{CF}_{4}$ molecule. Therefore, to calculate the cross sections for the transitions of groups II and IV, one should use in eqn. (4) the transition dipole moments of the $\nu_{4}$ and $\nu_{3}$ vibrational modes, respectively. The resulting cross section estimates are in reasonable agreement with the measured values. ${ }^{68}$ For example, the theoretical/experimental hard-sphere cross sections at $v_{\mathrm{E}}=11,15$, and 21 are $0.1 / 7,33 / 26$, and $20 / 6.5 \AA^{2}$ for group II transitions ( $\nu_{4}$ excitation), and 4/10, 110/42, and $100 / 19 \AA^{2}$ for group IV transitions ( $\nu_{3}$ excitation), respectively.

\subsection{Collisions with rare gas atoms ( $\mathrm{He}$ and $\mathrm{Ar}$ )}

3.2.1 Experimental observations. Measurements with $\mathrm{He}$ and $\mathrm{Ar}$ collision partners at low vibrational excitations $v_{\mathrm{E}}=0-2$ and $J_{\mathrm{E}}=55$ were performed at Swarthmore. ${ }^{71,72}$ The rate constants for $\mathrm{E} \rightarrow \mathrm{D}$ transition vary from 2 to $6.5 \times 10^{-11}$, being consistently larger for He. These values correspond to cross sections that are approximately equal for the two rare gases and do not exceed one tenth of the gas-kinetic 
cross section. The most striking difference with the collisions with $\mathrm{I}_{2}(\mathrm{X})$ is the observation of several additional fluorescence bands induced by CINAT. Particularly interesting is the fluorescence in the 338-344 $\mathrm{nm}$ region, assigned to the $\beta 1_{\mathrm{g}} \rightarrow \mathrm{A} 1_{\mathrm{u}}$ and $\mathrm{D}^{\prime} 22_{\mathrm{g}} \rightarrow \mathrm{A}^{\prime} 2_{\mathrm{u}}$ transitions. (A small contribution in this region from emission from the $\delta 2_{\mathrm{u}}$ state cannot be ruled out.)

The measured rate constants for all transitions are listed in Table 2 (together with their theoretical values discussed below) and are depicted in Fig. 4. The very similar efficiency of the CINAT to all three states is surprising. For $\mathrm{He}$, the $\mathrm{E} \rightarrow \mathrm{D}$ channel dominates at $v_{\mathrm{E}}=0$, whereas at $v_{\mathrm{E}}=2$ the combined contribution of the competing $\mathrm{E} \rightarrow \mathrm{D}^{\prime}$ and $\mathrm{E} \rightarrow \beta$ channels is larger. For $\mathrm{Ar}, \mathrm{E} \rightarrow \beta$ CINAT has the largest rate constant for $v_{\mathrm{E}}=0-2$.

Vibrational product state distributions for $v_{\mathrm{E}}=0$ and 2 are shown in Figs. 7-10. For $\mathrm{E} \rightarrow \mathrm{D}$ energy transfer, the distributions are fairly similar for $\mathrm{He}$ and Ar collision partners. As in the case of collisions with $\mathrm{I}_{2}(\mathrm{X})$, at $v_{\mathrm{E}}=0$ these distributions appear to be balanced between energy gap and FC effects. A more pronounced contribution of the near-resonant transition (to $v_{\mathrm{D}}=6$ ) can be seen when $v_{\mathrm{E}}=2$ is prepared, though the distributions resulting from both $\mathrm{He}$ and Ar collisions have peaks for D state levels with large FC factors. The distributions for the $\mathrm{D}^{\prime}$ and $\beta$ states are also similar for $\mathrm{He}$ and Ar. When $v_{\mathrm{E}}=0$ is prepared, the distributions are peaked at the lowest vibrational levels in accord with the FC factor distribution. When $v_{\mathrm{E}}=2$, the $\mathrm{D}^{\prime}$ state distributions resemble rather closely the distribution of $\mathrm{E}-\mathrm{D}^{\prime} \mathrm{FC}$ factors, much more so than the $\beta$ state distributions.

The CINAT from higher vibrational levels $v_{\mathrm{E}}=8-47$ was studied experimentally by the St. Petersburg group. ${ }^{66,68}$ The dependences of the total rate constants for the $\mathrm{E} \rightarrow \mathrm{D}$ transition at $J_{\mathrm{E}} \approx 55$ on $v_{\mathrm{E}}$ for $\mathrm{He}$ and $\mathrm{Ar}$ collision partners are presented in Fig. 4. The absolute values of the $k_{\mathrm{ED}}$ rate constant fall in the ranges $(2-8) \times 10^{-10}$ and $(6-20) \times 10^{-10}$ for $\mathrm{He}$ and $\mathrm{Ar}$, respectively, corresponding to very similar thermally averaged cross sections $\left(20-65 \AA^{2}\right)$, or $0.4-1$ of the gas-kinetic cross section $\sigma_{\mathrm{gk}}$. The variations of the rate constants with $v_{\mathrm{E}}$ are within the experimental error bars; on average, they gradually increase from $v_{\mathrm{E}}=8$ up to $v_{\mathrm{E}}=10-15$ and then remain almost constant. No dependence on the angular momentum $J_{\mathrm{E}}$ is observed.

Fig. 5 shows the vibrational product state distributions for selected initial vibrational excitations. The maxima roughly correspond to the near-resonant $v_{\mathrm{D}}$ vibrational levels, although in some cases are shifted towards lower levels by one or two vibrational quanta (e.g., $v_{\mathrm{E}}=8$ and $v_{\mathrm{E}}=20$ for Ar collisions). The distributions for Ar collisions are rather broad, covering 7 to 10 levels at half-maximum, and do not show any propensity for FC-like distributions. Teule et al. reported significantly narrower distributions with the same propensity to near-resonant transitions. ${ }^{64}$ In the present experiments, however, the spectral simulations considered the complete fluorescence band, whereas in ref. 64 only a limited spectral range was used to determine the distributions. Collisions with He produce less extended distributions, with a width at half maximum covering up to 7 levels.

Spectral simulations also make it possible to estimate the rotational product state distributions for the most populated $v_{\mathrm{D}}$ levels. In all cases they can be represented by a Gaussian profile with the maximum at $J_{\mathrm{D}}=J_{\mathrm{E}} \pm 1$ and half-width at half-maximum $\Delta J \approx \pm(7-10)$.

In addition to $\mathrm{D} \rightarrow \mathrm{X}$ fluorescence, three other fluorescence bands were observed for collisions with $\mathrm{Ar}$ (as well as with $\mathrm{N}_{2}$ and $\mathrm{CF}_{4}$ ). Features at $\lambda_{\max } \approx 342 \mathrm{~nm}$ and $\lambda_{\max } \approx 465 \mathrm{~nm}$ have been assigned to the $\delta 2_{\mathrm{u}} \rightarrow 2_{\mathrm{g}}(\mathrm{I}+\mathrm{I})$ and $\delta 2_{\mathrm{u}} \rightarrow 2_{\mathrm{g}}\left(\mathrm{I}+\mathrm{I}^{*}\right)$

Table 2 Total room temperature experimental and theoretical rate constants, in $\mathrm{cm}^{3}$ molecule ${ }^{-1} \mathrm{~s}^{-1}$, for the non-adiabatic $\mathrm{I}_{2}(\mathrm{E})+\mathrm{Rg}$ collisions The values in parentheses designate the power of ten. Theoretical rate constants are computed within the modified hard-sphere approximation, eqn. (18); accurate values computed by eqn. (16) are given in square brackets when available

\begin{tabular}{|c|c|c|c|c|c|}
\hline$V_{\mathrm{E}}$ & $n^{\prime}$ & EDW & $\operatorname{EVCC}(2 \times 2)$ & $\operatorname{EVCC}(6 \times 6)$ & Experiment \\
\hline \multicolumn{6}{|l|}{$\mathrm{He}$} \\
\hline \multirow[t]{3}{*}{0} & $\mathrm{D}$ & $2.4(-10)[7.9(-11)]$ & $1.5(-11)[1.6(-11)]$ & $1.5(-11)$ & $(3.8 \pm 0.5)(-11)$ \\
\hline & $\mathrm{D}^{\prime}$ & $2.3(-15)[5.9(-15)]$ & $1.3(-14)$ & $2.5(-12)$ & $(1.1 \pm 0.2)(-11)$ \\
\hline & $\beta$ & $1.7(-14)[2.2(-14)]$ & $1.5(-13)$ & $9.9(-13)$ & $(1.2 \pm 0.2)(-11)$ \\
\hline \multirow[t]{5}{*}{2} & $\mathrm{D}$ & $3.5(-10)[3.6(-10)]$ & $3.5(-11)[3.1(-11)]$ & $3.1(-11)$ & $(6.4 \pm 0.5)(-11)$ \\
\hline & $\mathrm{D}^{\prime}$ & $3.7(-15)[8.4(-15)]$ & $2.1(-14)$ & $3.6(-12)$ & $(4.7 \pm 1.8)(-11)$ \\
\hline & $\beta$ & $3.3(-14)[3.9(-14)]$ & $4.0(-13)$ & $1.8(-12)$ & $(4.8 \pm 1.9)(-11)$ \\
\hline & $\gamma$ & $8.9(-13)[1.0(-12)]$ & & $6.0(-13)$ & - \\
\hline & $\delta$ & $9.5(-13)[9.5(-13)]$ & & $3.1(-13)$ & - \\
\hline \multirow[t]{5}{*}{8} & $\mathrm{D}$ & {$[6.0(-10)]$} & $9.8(-11)[1.1(-10)]$ & $9.8(-11)$ & $1.9(-10)$ \\
\hline & $\mathrm{D}^{\prime}$ & {$[1.9(-14)]$} & $3.1(-14)$ & $5.1(-12)$ & - \\
\hline & $\beta$ & {$[1.2(-13)]$} & $7.8(-13)$ & $4.2(-12)$ & - \\
\hline & $\gamma$ & {$[2.6(-12)]$} & $8.8(-12)$ & $1.3(-12)$ & - \\
\hline & $\delta$ & {$[1.2(-11)]$} & $7.5(-11)$ & $9.3(-13)$ & - \\
\hline
\end{tabular}

\begin{tabular}{lll} 
Ar & & \\
\hline 0 & $\mathrm{D}$ & $2.4(-11)[2.8(-11)]$ \\
& $\mathrm{D}^{\prime}$ & $1.4(-17)[9.0(-16)]$ \\
& $\beta$ & $5.8(-17)[2.2(-16)]$ \\
& $\mathrm{D}$ & $1.4(-10)[1.7(-10)]$ \\
& $\mathrm{D}^{\prime}$ & $6.5(-17)[1.2(-15)]$ \\
& $\beta$ & $4.2(-16)[8.0(-16)]$ \\
& $\gamma$ & $6.3(-12)[6.6(-12)]$ \\
& $\delta$ & $4.2(-13)[4.5(-13)]$ \\
& $\mathrm{D}$ & {$[4.4(-10)]$} \\
& $\mathrm{D}$ & {$[5.7(-15)]$} \\
& $\beta$ & {$[1.4(-14)]$} \\
& $\gamma$ & {$[4.4(-11)]$} \\
& $\delta$ & {$[2.6(-10)]$}
\end{tabular}



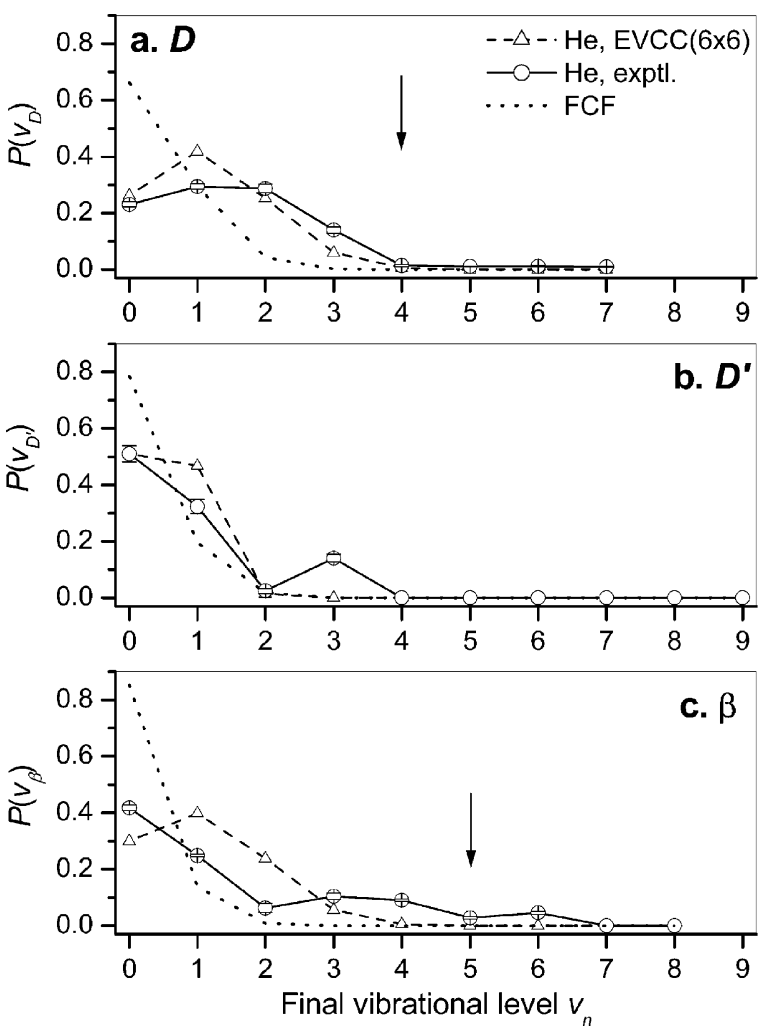

Fig. 7 Vibrational product state distributions for the $\mathrm{I}_{2}\left(\mathrm{E}, v_{\mathrm{E}}=0\right)+\mathrm{He}$ collisions in the final states $\mathrm{D}(\mathrm{a}), \mathrm{D}^{\prime}(\mathrm{b})$, and $\beta$ (c). Presented are experimental (circles) and theoretical EVCC (triangles) results, as well as and Franck-Condon factors (dashed lines). Arrows indicate the near-resonant levels. For the $\mathrm{D}^{\prime}$ state, the near-resonant level is $v_{\mathrm{D}^{\prime}}=10$.

transitions, respectively. ${ }^{68,76}$ The rate constant for $\mathrm{E} \rightarrow \delta$ CINAT is smaller than that for the $\mathrm{E} \rightarrow \mathrm{D}$ process (by an order of magnitude at $v_{\mathrm{E}}=9$ ). In turn, the transitions to the $\beta$ and $D^{\prime}$ states, if they exist, are at least ten times less efficient than
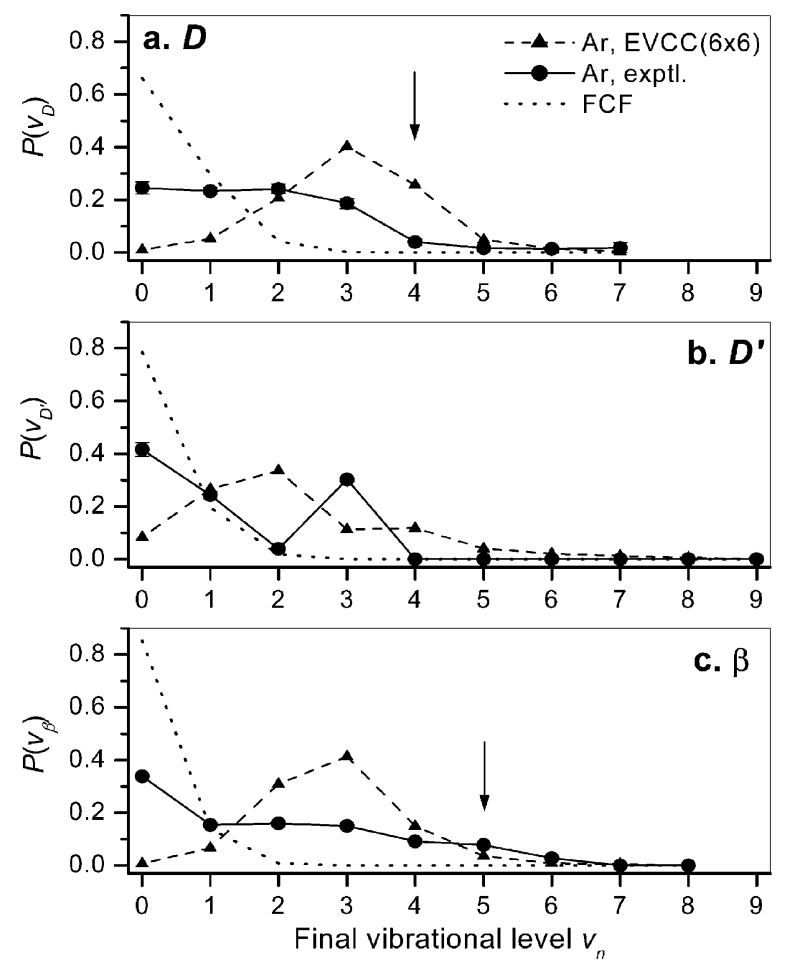

Fig. 8 Same as Fig. 7, but for $\mathrm{I}_{2}\left(\mathrm{E}, v_{\mathrm{E}}=0\right)+$ Ar collisions.
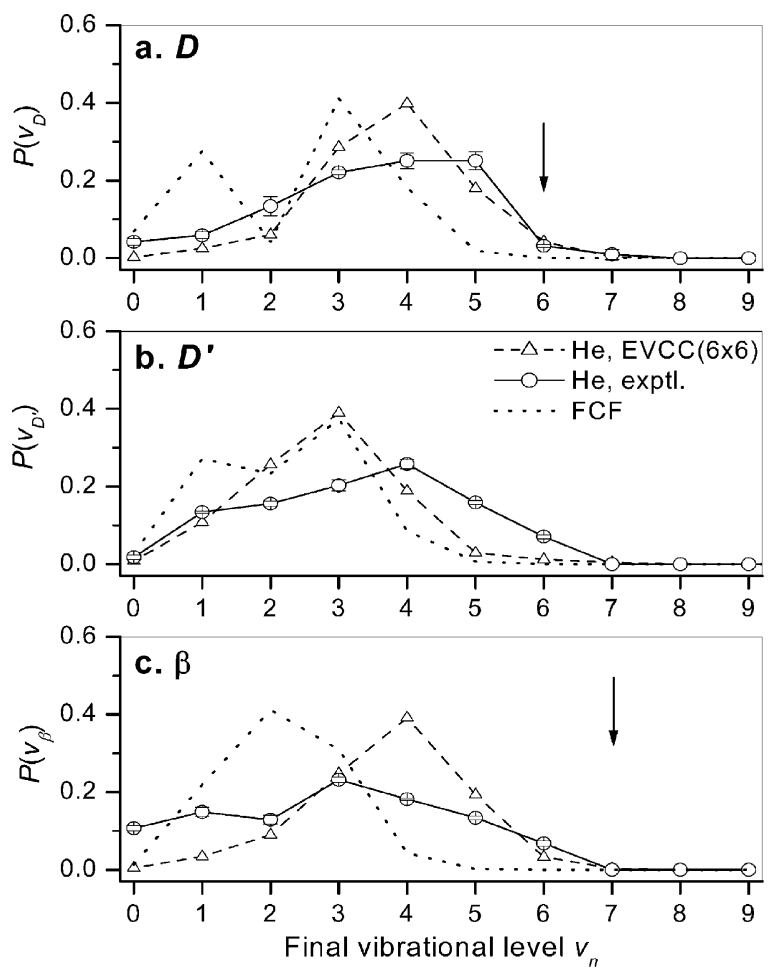

Fig. 9 Same as Fig. 7, but for the $\mathrm{I}_{2}\left(\mathrm{E}, v_{\mathrm{E}}=2\right)+\mathrm{He}$ collisions. For the $\mathrm{D}^{\prime}$ state, the near-resonant level is $v_{\mathrm{D}^{\prime}}=12$.

those to the $\delta$ state. Teule et al. $^{64}$ also observed the fluorescence at $342 \mathrm{~nm}$, but assigned it to the $\mathrm{D}^{\prime} \rightarrow \mathrm{A}^{\prime}$ transition.

It is clear that the transitions to the $\mathrm{D}^{\prime} 2_{\mathrm{g}}$ and $\beta 1_{\mathrm{g}}$ states, which have the same inversion parity as the initial $\mathrm{E} 0_{\mathrm{g}}^{+}$state, and to $\delta 2_{\mathrm{u}}$ with $\Delta \Omega=2$ cannot be understood within the electrostatic model proposed above for CINAT with molecular partners. As described below, an alternative theoretical
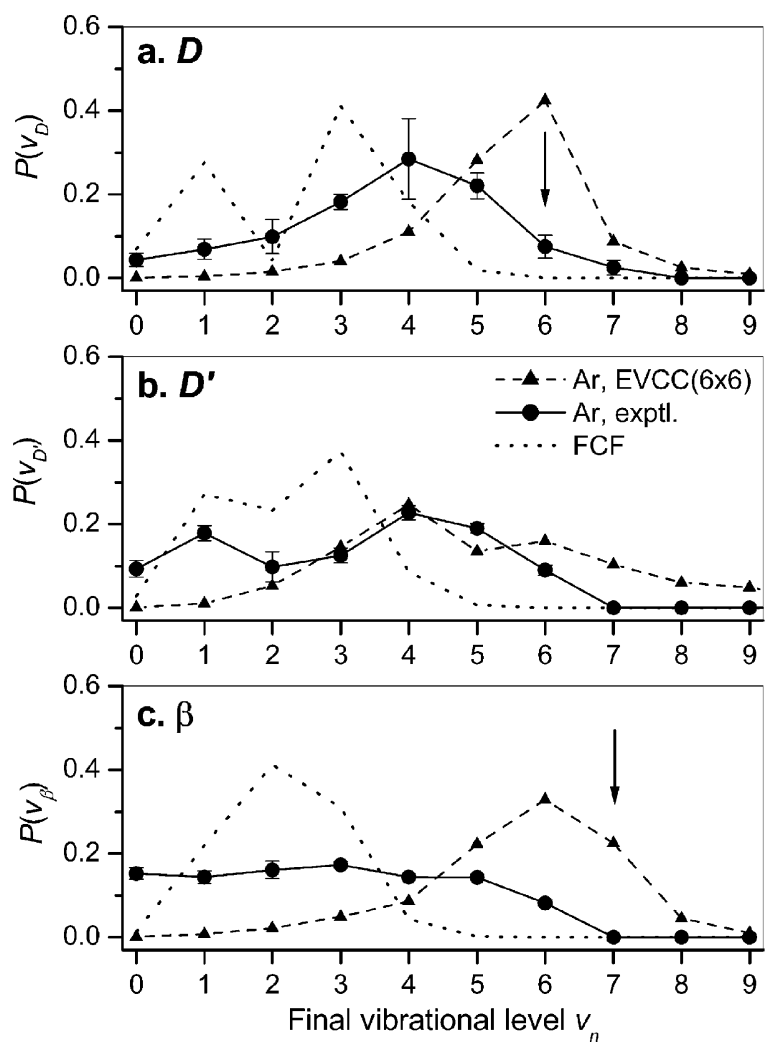

Fig. 10 Same as Fig. 9, but for the $I_{2}\left(E, v_{E}=2\right)+$ Ar collisions. 
approach is required, and has been recently pursued by the Moscow group.

3.2.2 Theoretical interpretation-IDIM PT1 model and quantum scattering theory. The framework for interpretation of the CINAT dynamics in collisions with rare gas $(\mathrm{Rg})$ atoms has been developed in refs. 73-75. It relies upon (i) the solution of the electronic structure problem for diabatic interaction potential energy surfaces (PESs) and coupling matrix elements within the intermolecular diatomics-in-molecule first-order perturbation theory (IDIM PT1) and (ii) implementation of decoupled dynamical approaches based on rigorous quantum scattering theory for a Hund case (c) diatomic molecule interacting with a structureless particle.

Like the electrostatic model, the IDIM PT1 model uses the asymptotic electronic wave functions (eqn. (2) and Table 1) to describe the IP states of the iodine molecule. However, in this case they are implemented within the diatomics-in-molecules (DIM) approach (see, e.g., refs. 106 and 107 as the zero-order wave functions for diabatic states of the system.

In brief, in the DIM method, the total electronic BornOppenheimer Hamiltonian is exactly decomposed into the sum of electronic Hamiltonians of all diatomic fragments of the system minus the redundant atomic Hamiltonians. Specifically, in the case of the $\mathrm{X}_{2}-\mathrm{Rg}$ interaction, one has

$$
\hat{H}_{\mathrm{el}}=\hat{H}_{\mathrm{X}_{\mathrm{A}} \mathrm{Rg}}+\hat{H}_{\mathrm{X}_{\mathrm{B}} \mathrm{Rg}}+\hat{H}_{\mathrm{X}_{2}}-\hat{H}_{\mathrm{X}_{\mathrm{A}}}-\hat{H}_{\mathrm{X}_{\mathrm{B}}}-\hat{H}_{\mathrm{Rg}},
$$

where $\alpha=\mathrm{A}, \mathrm{B}$ enumerates the $\mathrm{X}$ atoms. First-order DIM perturbation theory ${ }^{73,108,109}$ relates the zero-order approximation to the limit of infinitely separated fragments

$$
\hat{H}_{\mathrm{el}}^{0}=\hat{H}_{\mathrm{X}_{2}}+\hat{H}_{\mathrm{Rg}},
$$

whereas the perturbation term $\hat{V}=\hat{H}_{\mathrm{el}}-\hat{H}_{\mathrm{el}}^{0}$ is given by

$$
\hat{V}=\left[\hat{H}_{\mathrm{X}_{A} \mathrm{Rg}}-\hat{H}_{\mathrm{X}_{A}}-\hat{H}_{\mathrm{Rg}}\right]+\left[\hat{H}_{\mathrm{X}_{\mathrm{B}} \mathrm{Rg}}-\hat{H}_{\mathrm{X}_{\mathrm{B}}}-\hat{H}_{\mathrm{Rg}}\right] .
$$

Choosing zero-order wave functions as the product of electronic wave functions of the $\mathrm{X}_{2}$ molecule and of the closedshell $\mathrm{Rg}$ atom, one has, in the molecular-fixed frame related to vector $\hat{\boldsymbol{r}}$,

$$
\hat{H}_{\mathrm{el}}^{0}|n \Omega \sigma w\rangle|\mathrm{Rg}\rangle=w_{n \Omega \sigma w}|n \Omega \sigma w\rangle|\mathrm{Rg}\rangle,
$$

where $w_{n \Omega \sigma w}$ is the PEC of the corresponding IP state of the $\mathrm{X}_{2}$ molecule at the particular internuclear distance $r$. The constant electronic energy of $\mathrm{Rg}$ atom is dropped.

The perturbation matrix $\boldsymbol{V}$ in the basis set in eqn. (9) gives diabatic interaction PESs as the diagonal elements and coupling matrix elements as the non-diagonal ones. Using eqn. (2) to express the zero-order wave functions as the linear combinations of the products of three atomic functions, one obtains $^{108}$

$$
\boldsymbol{V}=\boldsymbol{C}^{+}\left[\boldsymbol{D}_{\mathrm{A}}{ }^{+} \boldsymbol{H}_{\mathrm{X}_{\mathrm{A}} \mathrm{Rg}} \boldsymbol{D}_{\mathrm{A}}+\boldsymbol{D}_{\mathrm{B}}{ }^{+} \boldsymbol{H}_{\mathrm{X}_{\mathrm{B}} \mathrm{Rg}} \boldsymbol{D}_{\mathrm{B}}-\boldsymbol{\varepsilon}_{\mathrm{A}} \otimes \mathbf{1}-\boldsymbol{\varepsilon}_{\mathrm{B}} \otimes \mathbf{1}\right] \boldsymbol{C},
$$

where the rotational matrices $\boldsymbol{D}_{\alpha}$, which transform the fragment Hamiltonians to a common frame related to $\hat{\boldsymbol{r}}$ diatomic axis, are composed from the Wigner rotational matrix elements $D_{m_{\alpha} m_{\alpha}^{\prime}}^{2 *}\left(0, \beta_{\alpha}, 0\right) .{ }^{110} \beta_{\alpha}$ is the angle between $\hat{\boldsymbol{r}}$ and $\hat{\boldsymbol{\rho}}_{\alpha}$ vectors, and $\mathbf{1}$ is a unit matrix. $\boldsymbol{C}$ is the matrix of the expansion coefficients in eqn. (2) and $\varepsilon_{\alpha}$ are the energies of iodine ions. The diatomic fragment matrices are expressed through the $\mathrm{Rg}-\mathrm{X}^{-}\left({ }^{1} \Sigma^{+}\right)$anion potential $V_{-}$and the $\mathrm{Rg}_{-}$ $\mathrm{X}^{+}\left({ }^{3} \Sigma^{-},{ }^{3} \Pi\right)$ cation potentials $V_{\Lambda}, \Lambda=\Sigma, \Pi$, at the particular $\mathrm{X}_{\alpha}-\mathrm{Rg}$ distance $\rho_{\alpha}$ using well-known formulas (see, e.g., refs. 111-113).

Eqn. (10) can be evaluated in analytical form. The list of formulas for the first-tier IP states is presented in ref. 73 For example, explicit expressions for $\mathrm{E} 0_{\mathrm{g}}^{+}-\mathrm{D} 0_{\mathrm{u}}^{+}$pair of states are

$$
\begin{aligned}
V_{\mathrm{EE}} & =V_{\mathrm{DD}}=v_{\mathrm{A}}+v_{\mathrm{B}}, \quad V_{\mathrm{ED}}=v_{\mathrm{A}}-v_{\mathrm{B}}, \\
v_{\alpha} & =\frac{1}{12}\left(5 V_{\Pi}^{\alpha}+V_{\Sigma}^{\alpha}\right)-\frac{1}{4}\left(V_{\Pi}^{\alpha}-V_{\Sigma}^{\alpha}\right) \cos ^{2} \beta_{\alpha}+\frac{1}{2} V_{\alpha},
\end{aligned}
$$

where $V_{\Lambda}{ }^{\alpha}=V_{\Lambda}\left(\rho_{\alpha}\right), V_{-}{ }^{\alpha}=V_{-}\left(\rho_{\alpha}\right)$. The couplings between the $\mathrm{E}$ and $\beta$ and $\mathrm{D}^{\prime}$ states are:

$$
\begin{gathered}
V_{E \beta}=v_{A}+v_{B}, \quad v_{\alpha}=-\frac{1}{4 \sqrt{3}}\left[\left(V_{\Pi}^{\alpha}-V_{\Sigma}^{\alpha}\right) \cos \beta_{\alpha} \sin \beta_{\alpha}\right], \\
V_{E D^{\prime}}=v_{A}+v_{B}, \quad v_{\alpha}=\frac{1}{4 \sqrt{3}}\left[\left(V_{\Pi}^{\alpha}-V_{\Sigma}^{\alpha}\right) \sin ^{2} \beta_{\alpha}\right],
\end{gathered}
$$

respectively. Alternative applications of the DIM-based approximations to IP states are considered in refs. 99,114

The IDIM PT1 model properly takes into account the symmetry of the problem thus providing the selection rules for electronic and rotational collision-induced transitions. These are displayed in Table 3 which shows the zeroes of the couplings at symmetric (linear and T-shaped) configurations and the parity of the coupling functions with respect to reflection in the plane which bisects the midpoint of diatomic axis. All the non-vanishing couplings between the $E$ state and other first-tier states are characterized. These symmetry properties are typical also for all other initial states and can be summarized as follows:

(1) Transitions between all states within the tier are allowed; electronic coupling matrix elements are symmetric and antisymmetric with respect to iodine nuclear inversion and provide selection rules $\Delta J=0, \pm 2, \ldots$ and $\Delta J= \pm 1, \pm 3, \ldots$ for transitions between the states of the same and opposite inversion parity $w$.

(2) The maximum couplings connect the states of same $j, \Omega$, and opposite inversion parity (as can be seen in eqns. (11)-(13) in these cases the couplings are magnified by the pairwise term proportional to $V_{-}^{73}$ )

To parameterize the DIM perturbation matrix, reliable interaction potentials of the $\mathrm{Rg}-\mathrm{I}^{ \pm}$ions must be obtained. Initially, the results of $a b$ initio averaged quadratic coupled cluster calulations (AQCC) was used to describe $\mathrm{I}_{2}-\mathrm{Ar}$ system. ${ }^{73-75}$ The new results presented in this paper are obtained with more accurate $\mathrm{Ar}-\mathrm{I}^{ \pm}$and $\mathrm{He}-\mathrm{I}^{ \pm}$potentials calculated by means of an unrestricted version of the coupled cluster method with single and double excitations and non-iterative correction to triple excitations (UCCSD[T]) from ref. 115. For the purpose of eliminating artificial induction interaction in the spirit of the "diatomics-in-ionic-system" model, ${ }^{116,117}$ all potentials are represented in the form

$$
V(\rho)=V_{\mathrm{sh}}(\rho)-f(\rho)\left(D_{4} / \rho^{4}+D_{6} / \rho^{6}+D_{8} / \rho^{8}\right),
$$

where $V_{\mathrm{sh}}$ is the short-range potential, $f$ is the switching function, and $D_{n}$ coefficients take into account long-range

Table 3 Symmetry properties of the diabatic couplings between the $E$ state and other first-tier IP states: zeroes at $C_{\infty \mathrm{v}}$ (linear) and $C_{2 \mathrm{v}}$ (T-shaped) configurations and parity

\begin{tabular}{lllll}
\hline Initial state & Final state & $C_{\infty \mathrm{v}}$ & $C_{2 \mathrm{v}}$ & Parity \\
\hline $\mathrm{E} 0_{\mathrm{g}}^{+}(j=2)$ & $\mathrm{D} 0_{\mathrm{u}}^{+}(j=2)$ & & 0 & - \\
& $\gamma 1_{\mathrm{u}}(j=2)$ & 0 & 0 & - \\
& $\delta 2_{\mathrm{u}}(j=2)$ & 0 & 0 & - \\
& $\beta 1_{\mathrm{g}}(j=2)$ & 0 & & + \\
& $\mathrm{D}^{\prime} 2_{\mathrm{g}}(j=2)$ & 0 & & + \\
\hline
\end{tabular}


dispersion and induction contributions. Modified potentials are

$$
V^{*}(\rho)=V_{\mathrm{sh}}(\rho)-f(\rho)\left(C_{6} / \rho^{6}+C_{8} / \rho^{8}\right),
$$

with $C_{n}$ dispersion coefficients obtained from $D_{n}$ ones as $C_{n}=D_{n}-\alpha_{n / 2-1} / 2 ; \alpha_{1}, \alpha_{2}$ and $\alpha_{3}$ are the lowest hyperpolarizabilities of the Rg atom. ${ }^{115}$

The diabatic interaction PESs and couplings from the IDIM PT1 model (together with spectroscopic PECs of the isolated iodine molecule) fully characterize the electronic part of the problem thus allowing us to use a consistent quantum scattering approach to the collision dynamics. Our implementation of the time-independent close coupling (CC) formalism for collisions of a diatomic molecule obeying Hund case (c) coupling scheme with a spherical structureless particle is presented in ref. 75. The $\mathrm{CC}$ equations describe the evolution of the system on the full or truncated set of coupled PESs and accurately take into account interaction of the electronic, vibrational, and rotational degrees of freedom.

The solution of the full system of CC equations for the processes under study is a formidable task. Due to the high density of the rovibronic states of the iodine molecule, the number of channels to be included into the $\mathrm{CC}$ basis set is very large. The use of the well-known infinite-order sudden (IOS) approximation $^{118}$ allows one to reduce greatly the dimension of the CC system by neglecting all rotational channels. It must be noted that the IOS approximation is expected to be valid for $\mathrm{I}_{2}+\mathrm{Rg}$ collisions, since they correspond to a light atom-heavy diatom system; ${ }^{118}$ for $\mathrm{I}_{2}+\mathrm{He}$ the applicability of the IOS approximation to vibrationally-inelastic collisions has been verified. ${ }^{119,120}$

The resulting method, EVCC-IOS ${ }^{75}$, keeps the close-coupling treatment of the electronic and vibrational channels. Further simplification can be achieved by the use of a perturbative treatment of the vibronic coupling in the spirit of Born approximation. ${ }^{121}$ A particularly appealing approach, which properly treats the zero-order scattered waves and preserves the unitarity of the $S$-matrix, is the exponential distorted wave (EDW) approximation. ${ }^{122}$ Under the EDW-IOS approximation, the transition probability is determined by a single potential matrix element which couples the initial and final vibronic levels. Therefore, EDW-IOS and EVCC-IOS (hereafter simply EDW and EVCC) can be regarded as the first- and infiniteorder methods for the coupling of vibronic scattering channels, respectively.

The formulas for the $S$-matrix elements and fixed-energy cross sections are given in ref. 75. To attain direct comparison with experimental data at room temperature, vibrationallyresolved and rotationally-summed rate constants for the the transitions between vibrational levels $v$ and $v^{\prime}$ of electronic states $n \Omega_{w}^{\sigma}, n^{\prime} \Omega_{w \prime}^{\sigma \prime}$ must be obtained by the standard formula:

$$
k_{n v, n^{\prime} v^{\prime}}=\left(\frac{8 \beta}{\pi \mu}\right)^{1 / 2} \int \beta E \sigma_{n v, n^{\prime} v^{\prime}}(E) \exp (-E \beta) \mathrm{d} E,
$$

where $\beta=1 / k_{\mathrm{B}} T, k_{\mathrm{B}}$ is the Boltzmann constant, and $T=300$ $\mathrm{K}$ for the present study. Calculation of the cross section energy dependence for evaluating converged rate constants is a computationally tractable task only for the EDW method and the EVCC method for two coupled PESs. In the EVCC calculations of higher dimensionality, the modified hard sphere (MHS) model is used. ${ }^{123}$ Assuming that

$$
\sigma(E) \approx \begin{cases}0, & \text { if } E \leq E_{0} \\ \sigma_{0}\left(1-E_{0} / E\right), & \text { if } E>E_{0}\end{cases}
$$

one gets from eqn. (16)

$$
k_{n v, n^{\prime} v^{\prime}}(\mathrm{MHS})=\left(\frac{8}{\pi \mu \beta}\right)^{1 / 2} \sigma_{0} \exp \left(-\beta E_{0}\right) .
$$

Despite the fact that the excitation transitions do not exhibit true threshold behavior, significant deviations occur only at relatively low collision energies. Test calculations show that the best estimates of the rate constants using eqn. (18) correspond to the $\sigma_{0}, E_{0}$ parameters determined from the cross sections computed at collision energies of 300 and $750 \mathrm{~cm}^{-1}$. The ratio of vibrationally-resolved to vibrationally-summed collision properties give the vibrational product state distributions.

It should be added that the IDIM PT1 model can be used in conjunction with less rigorous dynamical methods, for example, first-order semiclassical perturbation theory, ${ }^{74}$ an approach that is close to the semiclassical Born approximation for the electrostatic model discussed above.

The calculations were performed for collisions with $\mathrm{He}$ and Ar and initial excitations $v_{\mathrm{E}}=0,2$, and 8 at EDW and EVCC levels of theory. In the latter method different number of scattering channels can be included in the basis set. We will compare the results of the $\operatorname{EVCC}(2 \times 2)$ option, where only the initial $E$ and one particular final state are coupled, and almost converged EVCC $(6 \times 6)$ calculations which take into account the coupling between all six IP states of the first tier.

Total rate constants computed within the MHS model, eqn. (18), are presented in Table 2. As follows from the table, they reproduce reasonably well accurate values computed by eqn. (16) for $\mathrm{EDW}$ and $\operatorname{EVCC}(2 \times 2)$ providing that rate constant is not too small $\left(\geq 10^{-14}\right)$.

In all cases the EDW approximation clearly shows the propensity for transitions with changing inversion parity. The $\mathrm{E} \rightarrow \mathrm{D}$ transition is the most efficient. Rate constants for the transitions to the gerade states $\mathrm{D}^{\prime}$ and $\beta$ are almost two orders of magnitude lower than the transitions to ungerade states $\mathrm{D}$, $\gamma$, and $\delta$. Rate constants for the $\gamma$ and $\delta$ states at $v_{\mathrm{E}}=0$ (not shown) are also very small, but due to energetic, not symmetry, constrains-the corresponding scattering channels are practically inaccessible at thermal energies. The same propensity for inversion parity changing transitions has been found in the EDW calculations for $\mathrm{I}_{2}\left(\begin{array}{ll}\mathrm{D} & 0_{\mathrm{u}}^{+}\end{array}\right) \mathrm{CINAT}^{124}$ Qualitative analysis of the EDW results indicates that all non-adiabatic transitions occur at short range in the vicinity of the turning point of intermolecular potential.

Passing from the EDW to the $\operatorname{EVCC}(2 \times 2)$ level, one observes the reduction of the $\mathrm{E} \rightarrow \mathrm{D}$ rate constant by an order of magnitude and the growth of rate constants for other transitions. The rate constants for gerade states $\mathrm{D}^{\prime}$ and $\beta$ exhibit further increase in fully coupled $\operatorname{EVCC}(6 \times 6)$ calculations, a clear indication of an indirect nature of the corresponding transitions. As has been found in ref. 75 , it is the coupling to the $\mathrm{D} 0_{\mathrm{u}}^{+}$state which facilitates the population of gerade states. The mechanism can be interpreted as post-collision relaxation: the encounter of an iodine molecule with a rare gas atom induces the transitions to the ungerade states, specifically the $\mathrm{D}$ state. When the colliding partners fly apart, the D state undergoes further relaxation to the gerade states $\mathrm{D}^{\prime}$ and $\beta$. This indirect mechanism is quite efficient-the corresponding theoretical rate constants are only one order of magnitude less than the rate constant for the dominant "direct" $\mathrm{E} \rightarrow \mathrm{D}$ transition and comparable to those of transitions to $\gamma$ and $\delta$ states. Experimental measurements show that the relative efficiencies of indirect transitions are even higher.

The theoretical $\mathrm{E} \rightarrow \mathrm{D}$ rate constants rapidly grow with increasing $v_{\mathrm{E}}$ and provide reasonable matching between the sets of experimental data from the Swarthmore and St. Petersburg groups. For He, two other direct transitions to $\gamma$ and $\delta$ gain efficiency with similar rate, whereas the rate constants for indirect $\mathrm{E} \rightarrow \mathrm{D}^{\prime}$ and $\mathrm{E} \rightarrow \beta$ transitions increase more slowly. For Ar, however, the efficiencies of all transitions except $\mathrm{E} \rightarrow \gamma$ grow with the same rate. Actually, at $v_{\mathrm{E}}=8$, the calculations unambiguously reveal only the predominance of $\mathrm{E} \rightarrow \mathrm{D}$ CINAT and does not show any clear propensity for the population of other states. 
Quantitatively, the results of the $\operatorname{EVCC}(6 \times 6)$ calculations are in very good accord with experimental data for collisions with He. For collisions with Ar, larger difference between experiment and theory must be emphasized. The calculated rate constants at low $v_{\mathrm{E}}$ are too small with respect to the measured ones and those computed for He.

The vibrational product state distributions obtained from EVCC $(6 \times 6)$ MHS rate constants are compared with those measured experimentally in Figs. $7-10$ for $v_{\mathrm{E}}=0,2$ and in Fig. 5 for $v_{\mathrm{E}}=8$. For the $\mathrm{E} \rightarrow \mathrm{D}$ transition induced by $\mathrm{He}$, theory reproduces the experimental distributions very well and successfully describes their evolution from more FC-type to more resonant type as $v_{\mathrm{E}}$ increases. Agreement for the $\mathrm{E} \rightarrow \mathrm{D}^{\prime}, \beta$ transitions is not so perfect, although the theory is still able to mimic the main features of the distributions. The results for collisions with Ar are again worse, as can be seen in the plots for the $\mathrm{E} \rightarrow \mathrm{D}$ transition. Theory significantly overestimates the role of the energy gap between the initial and final vibrational levels; the calculated distributions are always peaked at the near-resonant energy levels. The calculated rotational product state distributions for a dominant $\mathrm{E} \rightarrow \mathrm{D}$ vibrational channel are peaked at $J_{\mathrm{D}}=J_{\mathrm{E}} \pm 1$ and cover at halfwidth four or five rotational levels, being virtually independent of initial vibrational excitation.

\section{Discussion and perspectives on future directions}

The experimental and theoretical results clearly identify two mechanisms for CINAT between the IP states of iodine molecule: "approach-induced" for collisions with molecular partners and more common "collision-induced" for collisions with rare gases.

The approach-induced mechanism manifests itself only in E $0_{\mathrm{g}}^{+} \rightarrow \mathrm{D} 0_{\mathrm{u}}^{+}$CINAT and gives large transition cross sections, including the giant ones that exceed the gas-kinetic cross in forty times. This process is highly selective: almost $90 \%$ of the products are formed in a single $v_{\mathrm{D}}$ vibrational level with a $\Delta J= \pm 1$ change in the angular momentum. The necessary conditions for this mechanism to occur are the existence of a final $v_{\mathrm{D}}$ level in a quasi-resonance with initially excited $v_{\mathrm{E}}$ level (ca. $|\Delta E| \leq 25 \mathrm{~cm}^{-1}$ ) and collisions with a particle possessing a permanent or transition electrostatic moment.

The features of the approach-induced mechanism can be qualitatively interpreted in the frame of an electrostatic model and the semiclassical Born approximation.

(i) The strong propensity for $\mathrm{E} 0_{\mathrm{g}}^{+} \rightarrow \mathrm{D} 0_{\mathrm{u}}^{+}$transition is a consequence of the giant transition dipole moment for $\Omega_{\mathrm{g}}^{\sigma} \leftrightarrow \Omega_{\mathrm{u}}^{\sigma}$ pair of states. It is much larger than the transition dipole moments for the states with different values of $\Omega$.

(ii) Being governed by the long-range electrostatic coupling, the non-adiabatic transitions occur at large interfragment distances. In this case transitions to near-resonant levels are strongly favored.

(iii) The low- $\Delta J$ propensity rule $\Delta J= \pm 1$ also follows from the selection rules for dipole-induced transitions.

The present interpretation disagrees with the predictions made by Lawley on the basis of the same electrostatic model, ${ }^{98}$ who found that the complex-formation (or orbiting-controlled) CINAT mechanism has cross sections that are only three times larger than the gas-kinetic cross sections. The long-range interaction was found to provide only a minor enhancement of the near-resonant complex-formation cross sections. While these estimates can be applicable to complexformation collisions, the far more important effect of the electrostatic coupling is the direct promotion of the non-adiabatic transitions at long distances.

The collisions with $\mathrm{I}_{2}(\mathrm{X})$ molecule provide the most striking example of the approach-induced CINAT. The sharp maxima in the rate constant dependence on the $\mathrm{E}$ state vibrational excitation (see Fig. 2) at $v_{\mathrm{E}}=14,32$, and 59 correspond to very small energy gaps $\left(\Delta E<1 \mathrm{~cm}^{-1}\right)$, and clearly illustrate the contribution of this mechanism.

For collisions with the $\mathrm{N}_{2}$ molecule, the probability of approach-induced transitions should be $c a .7$ times weaker due to the smaller quadrupole moment. The experimental data show similar features in the product state distributions, though the pronounced dependence of the rate constant on vibrational excitation in the E state is less clear, perhaps because of large error bars on the experimental measurements.

A different type of electrostatic interaction governs CINAT in collisions with the $\mathrm{CF}_{4}$ molecule. The $\mathrm{E} \rightarrow \mathrm{D}$ non-adiabatic coupling is facilitated by dipole-allowed excitations of the $\nu_{3}$ and $\nu_{4}$ vibrational modes in $\mathrm{CF}_{4}$. The corresponding transitions, however, exhibit only minor enhancement at the quasi-resonance conditions.

In summary, simple theoretical approaches in the spirit of first-order perturbation theory provide clear qualitative interpretation of experimental data on the approach-induced transitions. $^{68,69}$

Collisions with rare gas atoms represent a second mechanism for CINAT. For $\mathrm{E} \rightarrow \mathrm{D}$ energy transfer, much smaller rate constants and significantly broader product state distributions (vibrational and rotational) are observed. An interesting feature is the absence of strict selection rules for electronic transitions. While the $\mathrm{E} \rightarrow \mathrm{D}$ CINAT is still dominant, transitions to other IP states of the first tier are also efficient. Theoretical interpretation of CINAT in these systems is provided by quantum scattering calculations based on the realistic diabatic PESs and couplings calculated using the IDIM PT1 model.

This approach allows one to consider three levels of propensity rules. The "zero-order" level is given by the diabatic coupling matrix elements themselves. The IDIM PT1 model favors the $\mathrm{E} 0_{\mathrm{g}}^{+} \rightarrow \mathrm{D} 0_{\mathrm{u}}^{+}$transition. The EDW-IOS method sets the "first-order" level at which the transitions to different electronic states are considered as independent from each other. This level establishes the propensity for the transitions changing the inversion parity. The propensity rules of the third level are established by the close coupling treatment of the vibronic channels. It clearly indicates the importance of the post-collision, or indirect, non-adiabatic interactions, which proceed through an intermediate electronic state. The $\mathrm{E} 0_{\mathrm{g}}^{+}-\mathrm{D} 0_{\mathrm{u}}^{+}$ interaction efficiently populates the $\mathrm{D}$ state when the colliding partners closely approach each other. However, when they fly apart, the D state further relaxes via the transitions governed by the same "first-order" propensity rule for parity-changing transitions. This explains the relatively large population of the $\beta 1_{\mathrm{g}}$ and $\mathrm{D}^{\prime} 2_{\mathrm{g}}$ states observed at low vibrational excitations. Transitions to the $\gamma 1_{\mathrm{u}}$ and $\delta 2_{\mathrm{u}}$ states follow the direct mechanism being coupled directly to initial state $\mathrm{E}$.

Both the energy gap law and the Franck-Condon principle influence the vibrational product state distributions for the non-adiabatic transitions induced by collisions with rare gases. However, the latter factor plays a significant role at low vibrational excitations, $v_{\mathrm{E}}=0-2 .^{70-72}$ The first factor appears to be more important for CINAT at $v_{\mathrm{E}}>8$. For these high $v_{\mathrm{E}}$ levels, the vibrational product state distributions have an almost symmetric dumb-bell shape centered close to a near-resonant level, clear signature of the predominant role of the energy gap law. In general, the distributions of E-D FC factors are broader for higher values of $v_{\mathrm{E}}$ than is the case when $v_{\mathrm{E}}$ is low. For $v_{\mathrm{E}} \leq 2$, the near resonant $\mathrm{D}$-state vibrational levels are characterized by FC factors that are less than $4 \times 10^{-4}$, providing a significant vibrational overlap impediment to population of levels with small energy gaps. A universal model for the D-state vibrational distributions incorporating both energy gap and FC effects will necessarily appear to accentuate the importance of FC effects for these low values of $v_{\mathrm{E}}$. As the FC distribution broadens for higher values of $v_{\mathrm{E}}, \mathrm{FC}$ impediments become less severe. These considerations appear more conclusive in the 
case of the $\beta$ and $D^{\prime}$ state distributions observed following collisions of $\mathrm{I}_{2}\left(\mathrm{E}, v_{\mathrm{E}}=0-2\right)$ with $\mathrm{He}$ and Ar. The larger values of $\Delta T_{\mathrm{e}}$ for $\mathrm{E} \rightarrow \beta$ and $\mathrm{E} \rightarrow \mathrm{D}^{\prime}$ electronic energy transfer dictate that near-resonant transfer involves larger values of $\Delta v$ and correspondingly very small FC factors. All of the final state levels with non-negligible vibrational overlap involve large vibronic energy gaps. This effectively removes the latter consideration as means to direct vibrational populations, resulting in distributions that are dominated by $\mathrm{FC}$ considerations. It should be also noted that the indirect nature of these transitions may diminish the role of both the FC and near-resonant trends. Attempts to arrive at a more quantitative description of the vibrational product state distributions combining both trends have proven to be not yet successful. For example, an empirical formula by Katayama et al. ${ }^{100}$ fails to provide an unambiguous prediction, ${ }^{70}$ while the semiclassical model by Spalburg et al. ${ }^{125}$ is applicable only at high collision energies. ${ }^{74}$

Except for the lowest vibrational excitations, the rotational product state distributions obey the high- $\Delta J$ propensity rule, thus being much broader than those observed for approachinduced transitions. Rotational energy transfer can play a significant role compensating for the energy difference between the initial and final vibrational energy levels (see, e.g., ref. 68), which provides a rationale for why CINAT with rare gas collisions result in broad vibrational product state distributions.

Despite the remarkable progress in the interpretation of CINAT mechanisms, the experimental data still presents many challenges for experimental and theoretical studies.

For collisions with rare gases, CINAT that populates IP states other than $\mathrm{D}$ remains an ambiguous process. While the $\mathrm{E} 0_{\mathrm{g}}^{+} \rightarrow \beta 1_{\mathrm{g}}$ and $\mathrm{E} 0_{\mathrm{g}}^{+} \rightarrow \mathrm{D}^{\prime} 22_{\mathrm{g}}$ transitions are found to be very efficient at low vibrational excitations, ${ }^{71,72}$ transitions assigned to $\mathrm{E} 0_{\mathrm{g}}^{+} \rightarrow \delta 2_{\mathrm{u}}$ CINAT are prominent when higher $v_{\mathrm{E}}$ levels are initially excited. ${ }^{68}$ It would be instructive to perform new measurements to bridge the gap between these two sets of experimental data and to carry out more thorough studies of the weak fluorescence bands observed for $v_{\mathrm{E}}>8$. In its present form, the quantum scattering calculations successfully demonstrate the relatively high efficiency of direct and indirect CINAT processes, but is unlikely to be able to predict quantitatively the branching ratios between distinct channels and their variations with vibrational excitation. The present IDIM PT1 model certainly requires improvement. The model does not properly take into account electrostatic interactions, namely, the large polarizability of the IP states, ${ }^{68}$ related to a giant $\Omega_{\mathrm{g}}^{\sigma} \leftrightarrow \Omega_{\mathrm{u}}^{\sigma}$ transition dipole moment, which is responsible for very large approach-induced cross sections. Polarization forces created by the colliding partner may disturb the symmetric charge distribution of the bare iodine molecule and lead to significantly stronger interactions and non-adiabatic couplings. The fact that theoretical results for collisions with Ar are markedly worse than for collisions with $\mathrm{He}$ points out the importance of this effect.

For CINAT on molecular partners, a more sophisticated theoretical treatment of the approach-induced transitions is desirable, along with more precise measurements for different collision partners. The latter will be helpful in establishing the dependence of CINAT on the permanent and transition dipole and quadrupole moments of the collision partner. Collisions with the $\mathrm{I}_{2}(\mathrm{X})$ molecule reveal several particularly interesting features. At low vibrational excitations, the magnitudes of the rate constants, as well as the vibrational and rotational product state distributions, are quite similar to those observed following collisions with rare gas atoms, indicating a common short-range mechanism. At the same time, unlike the case of rare gas collisions, only the $\mathrm{D}$ state was found to be populated. Further, at higher levels of E state vibrational excitation, the non-resonant transitions have rate constants that are too large to be explained in such a model. It is not clear whether this enhancement is due to long-range electrostatic coupling or to complex formation, as suggested in refs. 67 and 98. Careful measurements of the rotational product state distributions and theoretical calculations incorporating both short- and long-range interactions, and appropriate treatment of the collision dynamics will help to answer this question. A very interesting issue is to investigate whether or not indirect CINAT processes to gerade IP states occur in collisions with molecular partners.

Fortunately, a number of experiments are possible to test the understanding of CINAT that we have deduced from the experiments and calculations applied to first tier electronic energy transfer in $I_{2}$. The experimental techniques used to explore dynamics in $I_{2}$ are directly applicable to a number of homo- and hetero-nuclear halogens. The ion-pair states in species such as $\mathrm{Br}_{2}$ and $\mathrm{Cl}_{2}$ share the basic energy level structure of the IP states of $\mathrm{I}_{2}$, though the dynamics of the lighter molecules may be more theoretically tractable, particularly from the standpoint of calculating accurate potential energy surfaces. Experiments performed at St. Petersburg on the $\mathrm{E} \rightarrow \mathrm{D}$ CINAT in $\mathrm{Cl}_{2}$ confirm the universal nature of the experimental approaches but reveal CINAT dynamics that differ remarkably from that discussed here for iodine molecule. ${ }^{77}$ Alternatively, the collision dynamics of species such as $\mathrm{IBr}$ and $\mathrm{ICl}$ are also experimentally accessible. These molecules offer an opportunity to explore the role of permanent dipole moments on the interaction potentials and dynamics. The heteronuclear diatomic halogens also exhibit a simplified electronic structure, as the lack of $\mathrm{g} / \mathrm{u}$ symmetry reduces the number of electronic states in each ion-pair tier from 6 to 3 .

Another very interesting opportunity to check the generality of the present interpretation of CINAT dynamics is to study the higher-lying IP states belonging to the second tier-correlating to $\mathrm{I}^{+}\left({ }^{3} \mathrm{P}_{j}\right)+\mathrm{I}^{-}\left({ }^{1} \mathrm{~S}_{0}\right)$ dissociation limits with $j=0$, 1 . In order of increasing $T_{\mathrm{e}}$, the six states belonging to the second tier are $\mathrm{f} 0_{\mathrm{g}}^{+}, \mathrm{g} 0_{\mathrm{g}}^{-}, \mathrm{F} 0_{\mathrm{u}}^{+}, \mathrm{G} 1_{\mathrm{g}}, \mathrm{H} 1_{\mathrm{u}}$, and $\mathrm{h} 0_{\mathrm{u}}^{-}$, see Fig. 1. The $f 0_{\mathrm{g}}^{+}$state is accessible for the same OODR excitation scheme through the B intermediate state. ${ }^{126,127}$ Results already obtained at St. Petersburg for collisions with $\mathrm{I}_{2}(\mathrm{X})$ at initial excitations $v_{\mathrm{f}}=8-19, J_{\mathrm{f}} \approx 55,85$ and 105 show a close similarity between the CINAT originating in the $\mathrm{f}$ and E states. ${ }^{69}$ As in the first tier, only $\Omega_{\mathrm{g}}^{\sigma} \leftrightarrow \Omega_{\mathrm{u}}^{\sigma} \mathrm{f} \rightarrow \mathrm{F}$ CINAT is observed and no transitions from the second to the first tier are detected. These findings are in accord with the electrostatic model. Quasi-resonant transitions dominate, with rate constants somewhat less than the quasi-resonant $\mathrm{E} \rightarrow \mathrm{D}$ rate constants. The low- $\Delta J$ propensity rule is valid for transitions with energy gaps $|\Delta E| \leq 25 \mathrm{~cm}^{-1}$. More detailed experimental and theoretical investigations will allow one to further explore the similarities and differences in the CINAT processes in the first and second tiers.

A particularly challenging future direction is the study of non-adiabatic transitions between IP states in van der Waals complexes. Such experiments may provide more detailed and direct information on the non-adiabatic dynamics than CINAT studies due to much more rigid constrains on available energy, angular momentum, and average orientation of the interacting particles. They will facilitate the analysis of the symmetry selection and propensity rules and provide spectroscopic data to directly test the theoretical PESs. There are indications that van der Waals complexes can be excited and provide fluorescence signals consistent with non-adiabatic transitions. Almost 15 years ago, Stephenson et al. observed such effects in the NeICl complex following excitation to the IP states. ${ }^{128-130}$ More recently, studies of IP states in large clusters formed in free expansions exhibited electronic energy transfer dynamics, ${ }^{23,131}$ consistent with the recent observation of emission assigned to the Rg complexes of $I_{2}(f)$ and $I_{2}(F)$ at St. Petersburg. ${ }^{132}$ The prospects for providing further insights into non-adiabatic dynamics with cluster-size and stateselective spectroscopic techniques are bright. 
Experimental and theoretical studies in some of these directions are currently underway in the Swarthmore, St. Petersburg, and Moscow groups.

\section{Acknowledgements}

We are grateful to the Editorial Board for providing the opportunity for stimulating discussions during the preparation of this work. Financial support to the Moscow and St. Petersburg groups by Russian Basic Research Fund (projects 02-0332676 and 02-03-32370), "Russian Universities" program (projects UR.05.03.013 and UR.01.01.050), and INTAS (9731573 and YS 03-55-1450) is gratefully acknowledged. The Swarthmore experimental program is supported by grants from the Camille and Henry Dreyfus Foundation and the Swarthmore College Faculty Research Fund.

\section{References}

1 J. F. Clarke and M. McChesney, Dynamics of Relaxing Gases, Butterfords, London, 1976.

2 W. B. DeMore, J. J. Margitan, M. J. Molina, R. T. Watson, D. H. Golden, R. F. Hampson, M. J. Kurilo, C. J. Howard and A. R. Raviskara, Chemical kinetics and photochemical data for use in stratospheric modeling, Evaluation No.7, JPL Pub. 85-37, Jet Propulsion Lab., Pasadena, CA, pp. 217.

3 R. D. Kenner and E. A. Ogryzlo, in Chemi- and Bioluminescence, ed. J. B. Burr, Marcel Dekker, New York, 1989, p. 45.

4 A. M. Pravilov, Photoprocesses in Molecular Gases, Energoatomizdat, Moscow, 1992, in Russian.

5 J. T. Yardley, Introduction to Molecular Energy Transfer, Academic Press, New York, 1980.

6 R. Bachman, X. Li, Ch. Ottinger, A. F. Vilesov and V. Wulfmeyer, J. Chem. Phys., 1993, 98, 8606.

7 N. Sadeghi and D. W. Setser, J. Chem. Phys., 1983, 79, 2710.

8 A. F. Vilesov, A. M. Pravilov and L. G. Smirnova, Sov. J. Chem. Phys., 1990, 7, 134.

9 P. J. Dagdigian, D. Patel-Misra, A. Berning, H.-J. Werner and M. H. Alexander, J. Chem. Phys., 1993, 98, 8580.

10 P. J. Dagdigian, Annu. Rev. Phys. Chem., 1997, 48, 95.

11 D. H. Katayama and A. V. Dentamaro, J. Chem. Phys., 1992, 97, 2820.

12 K. P. Huber and G. Herzberg, Molecular Spectra and Structure. IV. Constants of Diatomic Molecules, Van Nostrand-Reinhold, New York, 1979.

13 J. C. D. Brand and A. R. Hoy, Appl. Spectrosc. Rev., 1987, 23, 285.

14 K. P. Lawley and R. J. Donovan, J. Chem. Soc., Faraday Trans., 1993, 89, 1885.

15 M. E. Akopyan, N. K. Bibinov, D. B. Kokh, A. M. Pravilov and M. B. Stepanov, Chem. Phys., 1999, 242, 253.

16 K. Lawley, P. Jewsbury, T. Ridley, P. Langridge-Smith and R. Donovan, Mol. Phys., 1992, 75, 811.

17 H. Lefebvre-Brion and R. W. Field, Perturbations in the Spectra of Diatomic Molecules, Academic Press, Orlando, FL, 1986.

18 Excimer Lasers, ed. Ch. K. Rhodes, Springer, Berlin, 1984.

19 V. S. Zuev and L. D. Mikheev, Photochemical Lasers, Harwood Academic Publishers, Chur, 1991.

20 L. D. Mikheev, Quantum Electronics, 2002, 32, 1122.

21 J. Schroeder and J. Troe, Ann. Rev. Phys. Chem., 1987, 38, 163.

22 A. L. Harris, J. K. Brown and C. B. Harris, Ann. Rev. Phys. Chem., 1988, 39, 341.

23 S. Fei, X. Zheng, M. C. Heaven and J. Tellinghuisen, J. Chem. Phys., 1992, 97, 6057.

24 J. C. Alfano, D. Kliner, A. E. Johnson, N. E. Levinger and P. F. Barbara, in Ultrafast Phenomena VIII, ed. J. L. Martin, A. Migus, A. Mourou and A. H. Zewail, Springer, New York, 1992, p. 653.

25 M. Chergui and N. Schwentner, Trends Chem. Phys., 1992, 2, 89.

26 R. Zadoyan, M. Sterling and V. A. Apkaryan, J. Chem. Soc., Faraday Trans., 1996, 92, 1821.

27 R. Zadoyan, J. Alimy and V. A. Apkarian, Faraday Discuss., 1997, 108, 255.

28 V. A. Apkarian and N. Schwentner, Chem. Rev., 1999, 99, 1481.

29 C. Lienau and A. H. Zewail, J. Phys. Chem., 1996, 100, 18629.
30 J.-K. Wang, Q. Liu and A. H. Zewail, J. Phys. Chem., 1995, 99, 11309.

31 O. Z. Oldenberg, Z. Phys., 1924, 25, 136.

32 R. W. Wood, Philos. Mag., 1911, 21, 309.

33 J. Franck and R. W. Wood, Philos. Mag., 1911, 21, 314.

34 A. Elliot, Proc. R. Soc. London, Ser. A, 1940, 174, 273.

35 J. Tellinghuisen, Chem. Phys. Lett., 1974, 29, 359.

36 M. C. Sauer, Jr., W. A. Mulac, R. Cooper and F. Grieser, J. Chem. Phys., 1976, 64, 4587.

37 A. B. Callear and M. P. Metcalfe, Chem. Phys. Lett., 1976 , 43, 197.

38 A. B. Callear, P. Erman and J. Curepa, Chem. Phys. Lett., 1976, 44, 599.

39 Yu. Yu. Stoilov, Sov. J. Quantum Electron., 1978, 8, 223.

40 A. Guy, K. S. Viswanathan, A. Sur and J. Tellinghuisen, Chem. Phys. Lett., 1980, 73, 582.

41 H. Hemmati and G. J. Collins, Chem. Phys. Lett., 1980, 74, 488

42 V. N. Baboshin, L. D. Mikheev, A. B. Pavlov, V. P. Fokanov, M. A. Khodarkovskii and A. P. Shirokikh, Sov. J. Quantum Electron., 1981, 11, 683.

43 M. Martin, C. Fotakis, R. J. Donovan and M. J. Shaw, Nuovo Cimento Soc. Ital. Fis., B, 1981, 63, 300.

44 R. J. Donovan, B. V. O'Grady, L. Lain and C. Fotakis, J. Chem. Phys., 1983, 78, 3727 .

45 B. V. O'Grady and R. J. Donovan, Chem. Phys. Lett., 1985, 122, 503.

46 J. Tellinghuisen and L. F. Phillips, J. Phys. Chem., 1986, 90 , 5108 .

47 N. K. Bibinov and I. P. Vinogradov, Khim. Fiz., 1988, 7, 455 (in Russian).

48 M. V. McCusker, R. M. Hill, D. L. Huestis, D. C. Lorents, R. A Gutcheck and H. H. Nakano, Appl. Phys. Lett., 1975, 27, 363.

49 R. S. Bradford, E. R. Ault and M. L. Bhaumik, Appl. Phys. Lett., 1975, 27, 546.

50 J. J. Ewing and C. A. Brau, Appl. Phys. Lett., 1975, 27, 557.

51 L. D. Mikheev, A. P. Shirokikh, A. V. Startsev and V. S. Zuev, Opt. Commun., 1978, 26, 237.

52 V. S. Zuev, L. D. Mikheev, A. V. Startsev and A. P. Shirokikh, Sov. J. Quant. Electron., 1979, 9, 1195

53 M. J. Shaw, C. B. Edwards, F. O'Neil, C. Fotakis and R. J. Donovan, Appl. Phys. Lett., 1980, 37, 346.

54 K. Watanabe, S. Kashivabara and R. Fujimoto, Appl. Phys. Lett., 1987, 50, 629.

55 J. I. Steinfeld, J. Phys. Chem. Ref. Data, 1984, 13, 445.

56 J. Tellinghuisen, S. Fei, X. Zheng and M. C. Heaven, Chem. Phys. Lett., 1991, 176, 373.

57 D. L. Rousseau and P. F. Williams, Phys. Rev. Lett., 1974, 33 1368.

58 J. B. Koffend, A. M. Sibai and R. Bacis, J. Phys., 1982, 43, 1639

59 Z. Min, C. Li, J. Wang and S. Gong, Chem. Phys. Lett., 1992, 196, 139.

60 J. P. Perrot, M. Broyer, J. Chevaleyre and B. Femelat, J. Mol Spectrosc., 1983, 98, 161

61 M. D. Danyluk and G. W. King, Chem. Phys., 1977, 25, 343.

62 W. Ubachs, I. Aben, J. B. Milan, G. S. Somsen, A. G. Stuiver and W. Hogervorst, Chem. Phys., 1993, 174, 285.

63 D. Inard, D. Cerny, M. Nota, R. Bacis, S. Churassy and V. Skorokhodov, Chem. Phys., 1999, 243, 305.

64 R. Teule, S. Stolte, W. Ubachs, I. Aben, J. B. Milan, G. S Somsen, A. G. Stuiver and W. Hogervorst, Laser Chem., 1999, 18, 111 .

65 M. E. Akopyan, N. K. Bibinov, D. B. Kokh, A. M. Pravilov, M. B. Stepanov and O. S. Vasyutinskii, Chem. Phys., 1999, 242, 263.

66 M. E. Akopyan, N. K. Bibinov, D. B. Kokh, A. M. Pravilov, O. L. Sharova and M. B. Stepanov, Chem. Phys., 2001, 263, 459.

67 N. K. Bibinov, O. L. Malinina, A. M. Pravilov, M. B. Stepanov and A. A. Zakharova, Chem. Phys., 2002, 277, 179.

68 M. E. Akopyan, A. M. Pravilov, M. B. Stepanov and A. A Zakharova, Chem. Phys., 2003, 287, 399.

69 M. E. Akopyan, I. Yu. Chinkova, T. V. Fedorova, S. A Poretsky and A. M. Pravilov, Chem. Phys., accepted.

70 C. J. Fecko, M. A. Freedman and T. A. Stephenson, J. Chem. Phys., 2001, 115, 4132.

71 C. J. Fecko, M. A. Freedman and T. A. Stephenson, J. Chem. Phys., 2002, 116, 1361.

72 P. P. Chandra and T. A. Stephenson, J. Chem. Phys., submitted.

73 T. V. Tscherbul, A. V. Zaitsevskii, A. A. Buchachenko and N. F. Stepanov, Russ. J. Phys. Chem., 2003, 77, 511.

74 T. V. Tscherbul and A. A. Buchachenko, Chem. Phys. Lett. 2003, 370, 563 . 
75 T. V. Tscherbul and A. A. Buchachenko, J. Phys. B, 2004, 37, 1605.

76 M. E. Akopyan, A. M. Pravilov, M. B. Stepanov and A. A. Zakharova, J. Phys. B, 2003, 36, 2873.

77 N. K. Bibinov, M. A. Nikitin, A. M. Pravilov and A. A Zakharova, Chem. Phys., 2002, 277, 191.

78 R. J. LeRoy, University of Waterloo Chemical Physics Research Report, No.CP-230R3, University of Waterloo, Canada, 1986.

79 J. C. D. Brand, A. R. Hoy, A. K. Kalkar and A. B. Yamashita, J. Mol. Spectrosc., 1982, 95, 350.

80 T. Ishiwata and I. Tanaka, Laser Chem., 1987, 7, 79

81 R. F. Barrow and K. K. Yee, J. Chem. Soc.,Faraday Trans. 2, 1973, 69, 684.

82 T. Ishiwata, T. Kusayanagi, T. Hara and I. Tanaka, J. Mol. Spectrosc., 1986, 119, 337.

83 T. Ishiwata, T. Hara, K. Obi and I. Tanaka, J. Chem. Phys., 1987, 87, 2513

84 T. Ishiwata, S. Motohiro, E. Kagi, H. Fujiwara and M. Fukushima, Bull. Chem. Soc. Japan, 2000, 73, 2255.

85 S. Gersternkorn and P. Luc, J. Phys. (Paris), 1985, 46, 867.

86 E. Kagi, N. Yamamoto, H. Fujiwara, M. Fukushima and T. Ishiwata, J. Mol. Spectr., 2002, 216, 48

87 S. Motohiro, S. Nakajima and T. Ishiwata, J. Chem. Phys., 2002, 117, 187.

88 S. Motohiro, S. Nakajima, K. Aoyama, E. Kagi, H. Fujiwara and M. Fukushima, J. Chem. Phys., 2002, 117, 9777.

89 S. Motohiro, S. Nakajima and T. Ishiwata, J. Mol. Spectrosc., 2002, 212, 194.

90 M. L. Nowlin and M. C. Heaven, Chem. Phys. Lett., 1995, 239, 1.

91 J. P. Perrot, B. Femelat, J. L. Subtil, M. Broyer and J. Chevaleyre, Mol. Phys., 1987, 61, 85.

92 P. J. Wilson, T. Ridley, K. P. Lawley and R. J. Donovan, Chem. Phys., 1994, 182, 325.

93 P. Jewsbury and K. Lawley, Chem. Phys., 1990, 141, 225.

94 R. S. Mulliken, J. Chem. Phys., 1971, 55, 288.

95 J. Li and K. Balasubramanian, J. Mol. Spectrosc., 1989, 138, 162.

96 W. A. de Jong, L. Visscher and W. C. Nieuwpoort, J. Chem. Phys., 1997, 107, 9046.

97 W. Moffit, Proc. R. Soc. London, Sect. A, 1951, 210, 245.

98 K. Lawley, Chem. Phys., 1988, 127, 363.

99 V. S. Batista and D. F. Coker, J. Chem. Phys., 1997, 106, 6923.

100 D. H. Katayama, T. A. Miller and V. E. Bondybey, J. Chem. Phys., 1979, 71, 1662.

101 J. Derouard and N. Sadeghi, Chem. Phys. Lett., 1984, 111, 353.

102 M. H. Alexander, J. Chem. Phys., 1982, 76, 429.

103 M. H. Alexander, A. Berning, A. D. Esposti, A. Joerg, A Kliesch and H.-J. Werner, Ber. Bunsenges. Phys. Chem., 1990, 94, 1253.

104 A. N. Dvoryankin, L. B. Ibragimova, Yu. A. Kulagin and L. A. Shelepin, in Plasma Chemistry, ed. B. M. Smirnov, No.14, Energoatomizdat, Moscow, 1987, p. 102, (in Russian).
105 D. B. Kokh, A. B. Alekseev and R. J. Buenker, personal communication.

106 J. C. Tully, in Semiempirical Methods in Electronic Structure Theory, ed. G. A. Segal, Pt. A, Plenum, N. Y., 1977.

107 P. J. Kunz, in Atom-Molecule Collision Theory, ed. R. B. Bernstein, Plenum, N. Y., 1979, p. 79

108 A. A. Buchachenko and N. F. Stepanov, J. Chem. Phys., 1996 104, 9913.

109 A. A. Buchachenko and N. F. Stepanov, Russ. J. Phys. Chem., 1998, 72, 61.

110 R. N. Zare, AngularMomentum, Wiley, New York, 1988.

111 V. Aquilanti and P. Grossi, J. Chem. Phys., 1980, 73, 1165.

112 Z. Ma, K. Liu, L. B. Harding, M. Komotos and G. C. Schatz, J. Chem. Phys., 1994, 100, 8026.

113 R. V. Krems and A. A. Buchachenko, J. Phys. B, 2000 , 33, 4551 .

114 B. L. Grigorenko, A. V. Nemukhin and N. V. Ozhegova, Chem. Phys. Lett., 1998, 296.

115 A. A. Buchachenko, T. V. Tscherbul, J. Klos, M. M. Szczesniak and G. Chalasinski, Mol. Phys., submitted.

116 I. Last and T. F. George, J. Chem. Phys., 1987, 87, 1183

117 I. Last and T. F. George, J. Chem. Phys., 1988, 89, 3071

118 D. J. Kouri, in Atom-Molecule Collision Theory, ed. R. B. Bernstein, Plenum, New York, 1979, p. 301.

119 S. Eggert, S. Tellez and G. A. Pfeffer, J. Phys. Chem., 1987, 91 1414.

120 G. A. Pfeffer, J. Phys. Chem., 1987, 91, 2808.

121 L. D. Landau and E. M. Lifshitz, Quantum Mechanics, AddisonWesley, Reading, 1958.

122 G. G. Balint-Kurti, Int. Rev. Sci., 1975, 1, 286.

123 G. G. Chernyi, S. A. Losev, S. O. Merchet, B. V. Potapkin, Physical and Chemical Processes in Gas Dynamics: Cross Sections and Rate Constants, AIAA, Reston, 2002, vol. 1.

124 T. V. Tscherbul and A. A. Buchachenko, Khim. Fiz., accepted (in Russian).

125 M. R. Spalburg, J. Los and E. A. Gislason, Chem. Phys., 1985, 94, 327.

126 U. Heemann, H. Knoeckel and E. Tiemann, Chem. Phys. Lett., 1982, 90, 17

127 R. J. Donovan, A. J. Holmes, P. R. R. Langridge-Smith and T. Ridley, J. Chem. Soc., Faraday Trans., 1988, 84, 541.

128 T. A. Stephenson, Y. Hong and M. I. Lester, Chem. Phys. Lett., $1989,159,549$.

129 T. A. Stephenson, Y. Hong and M. I. Lester, in Dynamics of Polyatomic van der Waals Complexes, ed. N. Halberstadt and K. C. Janda, Plenum, New York, 1990, p. 493.

130 T. A. Stephenson, Y. Hong and M. I. Lester, J. Chem. Phys., 1991, 94, 4171.

131 K. L. Randall and D. J. Donaldson, Chem. Phys., 1996 , 211, 377.

132 M. E. Akopyan, I. Yu. Novikova, S. A. Poretsky and A. M. Pravilov, in preparation. 